Pacific

Journal of

Mathematics

\title{
RESTRICTED LIMITS OF MINIMAL AFFINIZATIONS
}

ADRIANO MOURA 


\title{
RESTRICTED LIMITS OF MINIMAL AFFINIZATIONS
}

\begin{abstract}
ADRIANO MOURA
We obtain character formulas of minimal affinizations of representations of quantum groups when the underlying simple Lie algebra is orthogonal and the support of the highest weight is contained in the first three nodes of the Dynkin diagram. We also give a framework for extending our techniques to a more general situation. In particular, for the orthogonal algebras and a highest weight supported in at most one spin node, we realize the restricted classical limit of the corresponding minimal affinizations as a quotient of a module given by generators and relations and, furthermore, show that it projects onto the submodule generated by the top weight space of the tensor product of appropriate restricted Kirillov-Reshetikhin modules. We also prove a conjecture of Chari and Pressley regarding the equivalence of certain minimal affinizations in type $D_{4}$.
\end{abstract}

\section{Introduction}

The representation theory of affine Kac-Moody algebras and their quantum groups has been intensively studied from a broad range of perspectives in the last two decades. In this paper, we focus on nontwisted quantum affine Kac-Moody algebras and their finite-dimensional representations. Let $\mathfrak{g}$ be a finite-dimensional simple Lie algebra over the complex numbers, let $\tilde{\mathfrak{g}}=\mathfrak{g} \otimes \mathbb{C}\left[t, t^{-1}\right]$ be the associated loop algebra, and let $U_{q}(\mathfrak{g})$ and $U_{q}(\tilde{\mathfrak{g}})$ be their Drinfeld-Jimbo quantum groups over $\mathbb{C}(q)$, where $q$ is an indeterminate. The affine Kac-Moody algebra is a one-dimensional central extension of $\tilde{\mathfrak{g}}$, but since the center acts trivially on finite-dimensional modules, it suffices to consider the loop algebra. It turns out that the finite-dimensional representations of $U_{q}(\tilde{\mathfrak{g}})$ are $\ell$-weight modules, that is, every vector is a linear combination of common generalized eigenvectors for $U_{q}(\tilde{\mathfrak{h}})$, where $\mathfrak{h}$ is a fixed Cartan subalgebra of $\mathfrak{g}$ and $\tilde{\mathfrak{h}}=\mathfrak{h} \otimes \mathbb{C}\left[t, t^{-1}\right]$. Moreover, the simple modules are highest- $\ell$-weight and the set of all dominant $\ell$-weights is in bijection with the monoid $\mathscr{P}_{q}^{+}$of $n$-tuples of polynomials in one variable with constant term 1 , where $n$ is the rank of $\mathfrak{g}$. The set of all $\ell$-weights corresponds

MSC2000: primary 17B10, 17B37, 17B67; secondary 17B65, 81R10, $17 \mathrm{~B} 70$.

Keywords: minimal affinizations, Kirillov-Reshetikhin modules, quantum affine algebras, representation theory, characters.

This work was partially supported by CNPq and FAPESP. 
to the group $\mathscr{P}_{q}$ associated to $\mathscr{P}_{q}^{+}$. By specializing $q$ at 1 , one recovers the finitedimensional representation theory of $\tilde{\mathfrak{g}}$.

Given a nonzero complex number $a$, let $\mathrm{ev}_{a}: \tilde{\mathfrak{g}} \rightarrow \mathfrak{g}$ be the evaluation map $x \otimes f(t) \mapsto f(a) x$. If $V$ is a $\mathfrak{g}$-module, one can consider the pullback $V(a)$ of $V$ by $\mathrm{ev}_{a}$. In particular, every irreducible $\mathfrak{g}$-module can be turned into a $\tilde{\mathfrak{g}}$-module. In the quantum case, unless $\mathfrak{g}$ is of type $A$, there is no analogue of the evaluation map, and in fact, most often an irreducible $U_{q}(\mathfrak{g})$-module cannot be turned into a $U_{q}(\tilde{\mathfrak{g}})$-module. By allowing the underlying vector space to be enlarged in a "controlled" way, a concept of quantum affinization of an irreducible $U_{q}(\mathfrak{g})$-module was introduced in [Chari 1995]. Two affinizations are said to be equivalent if they have isomorphic $U_{q}(\mathfrak{g})$-structures. It follows from the classification of the finitedimensional irreducible $U_{q}(\tilde{\mathfrak{g}})$-modules that every finite-dimensional irreducible $U_{q}(\mathfrak{g})$-module has at least one equivalence class of affinizations. Moreover, there are finitely many equivalence classes of affinizations, and the usual partial order on the weight lattice $P$ of $\mathfrak{g}$ induces a partial order on the set of equivalence classes of affinizations of a given irreducible $U_{q}(\mathfrak{g})$-module. Representatives of the minimal elements with respect to this partial order are called minimal affinizations. Although an almost complete classification of the highest $\ell$-weights of equivalence classes of minimal affinizations was obtained by Chari [1995] and by Chari and Pressley [CP 1996b; 1995; 1996a], their structure remained essentially unknown except when $\mathfrak{g}$ is of type $A$ or $B_{2}$. Further progress was made after the introduction of the concept of $q$-characters in [Frenkel and Reshetikhin 1999], which we prefer to call $\ell$-characters as explained in Section 4.8.

The $\ell$-character of a finite-dimensional $U_{q}(\tilde{\mathfrak{g}})$-module $V$ is the associated element $\operatorname{char}_{\ell}(V)$ of the integral group ring $\mathbb{Z}\left[\mathscr{P}_{q}\right]$ that records the dimensions of the $\ell$-weight spaces of $V$. Given $\lambda \in \mathscr{P}_{q}^{+}$, let us denote by $V_{q}(\lambda)$ the irreducible $U_{q}(\tilde{\mathfrak{g}})$-module with highest $\ell$-weight $\lambda$. Finding formulas for the $\ell$-character of $V_{q}(\lambda)$ is still an open problem in general. In [2001], E. Frenkel and E. Mukhin defined an algorithm, now widely known as the Frenkel-Mukhin algorithm, which for a given $\lambda \in \mathscr{P}_{q}^{+}$returns an element of $\mathbb{Z}\left[\mathscr{P}_{q}\right]$ that was conjectured and proved in certain situations to be $\operatorname{char}_{\ell}\left(V_{q}(\lambda)\right)$. Nakai and Nakanishi [2008] recently showed this is not always the case. However, even in the situations for which the conjecture holds, translating the information given by the algorithm into general closed formulas remains a challenge. For further details on the theory of $\ell$-characters, beside the aforementioned literature, see the survey [Chari and Hernandez 2008] and its references. We remark that Nakai and Nakanishi [2007b; 2007a] give pathtableaux descriptions of Jacobi-Trudi determinants that, conjecturally, coincide with the $\ell$-characters if $\mathfrak{g}$ is of classical type. Hernandez [2007] partially proved this conjecture if $\mathfrak{g}$ is of type $B$; see also [Chari and Hernandez 2008]. 
Another approach for studying minimal affinizations is to consider their classical limit. Even though most of the $\ell$-character information is lost, this process provides an effective tool for studying their $U_{q}(\mathfrak{g})$-structure, that is, their characters. The $U_{q}(\mathfrak{g})$-structure of the minimal affinizations belonging to the family of Kirillov-Reshetikhin modules was obtained in [Chari 2001] partially using this approach. The proof consisted in showing that the conjectural character was both a lower and an upper bound for the character of the given Kirillov-Reshetikhin module. While the latter was proved by working with the classical limit, the proof of the former was done in the quantum context. Later on, Chari and Moura [CM 2006; 2007] showed that both "upper and lower bound" parts of the proofs of the results of [Chari 2001] could be performed by working with the current algebra $\mathfrak{g}[t]=\mathfrak{g} \otimes \mathbb{C}[t]$; they also obtained in this manner characters of KirillovReshetikhin modules for twisted affine algebras. These modules were introduced in [Kirillov and Reshetikhin 1987] (in the context of Yangians rather than quantum affine algebras) in connection with the Bethe Ansatz. They are the minimal affinizations of the irreducible $U_{q}(\mathfrak{g})$-modules whose highest weights are multiples of the fundamental weights of $\mathfrak{g}$.

The main goal of this paper is to initiate a program for extending the approach of [CM 2006; 2007] to more general minimal affinizations other than KirillovReshetikhin modules. We prove several partial results in this direction and carry out the whole program in the simplest cases. In particular, we obtain character formulas for minimal affinizations in the case that $\mathfrak{g}$ is orthogonal and the support of the highest weigh is contained in the first three nodes of the Dynkin diagram of $\mathfrak{g}$. We now give a summary of our results.

Given a dominant integral weight $\lambda=\sum m_{i} \omega_{i}$ (where $\omega_{i}$ for $i=1, \ldots, n$ are the fundamental weights of $\mathfrak{g}$ ), we define restricted graded $\mathfrak{g}[t]$-modules $M(\lambda)$ and $T(\lambda)$. The former is given by generators and relations, while the latter is the submodule generated by the top weight space of $\otimes_{i} M\left(m_{i} \omega_{i}\right)$. We conjecture that these modules are isomorphic. This is a generalization of one of the main results of [CM 2006; 2007]. The conjecture clearly holds for type $A$. The defining relations for the module $M(\lambda)$ are, roughly speaking, the intersection of the relations satisfied by the corresponding restricted Kirillov-Reshetikhin modules $M\left(m_{i} \omega_{i}\right)$. In particular, it is immediate that $T(\lambda)$ is a quotient of $M(\lambda)$. We prove this conjecture when $\mathfrak{g}$ is orthogonal and $\lambda$ is supported only in the first three nodes of the Dynkin diagram of $\mathfrak{g}$. If $\mathfrak{g}$ is of type $D$, the proof also works in the case that both spin nodes are in the support of $\lambda$. As a byproduct of the proof, we obtain the characters of the modules $M(\lambda)$ in these cases. Namely, assume $\mathfrak{g}$ is of type $B_{n}$ and that the nodes of the Dynkin diagram of $\mathfrak{g}$ are labeled as in [Humphreys 1972]. Given $\lambda=m_{1} \omega_{1}+m_{2} \omega_{2}+m_{3} \omega_{3}$, consider the set

$$
\mathscr{A}=\left\{\boldsymbol{r}=\left(r_{1}, r_{2}, r_{3}\right) \in \mathbb{Z}_{\geq 0}^{3}: r_{1}+r_{2} \leq\left[a_{3} m_{3}\right], r_{2} \leq m_{1}, r_{3} \leq\left[a_{2} m_{2}\right]\right\},
$$


where $[m]$ denotes the integer part of the rational number $m, a_{n}=1 / 2$, and $a_{i}=1$ for $i \neq n$. Then, we have an isomorphism of $\mathfrak{g}$-modules:

$$
\text { (1) } M(\lambda) \cong \bigoplus_{r \in \mathscr{A}} V\left(\left(m_{1}+r_{1}-r_{2}\right) \omega_{1}+\left(m_{2}+r_{2}-a_{2}^{-1} r_{3}\right) \omega_{2}+\left(m_{3}-a_{3}^{-1}\left(r_{1}+r_{2}\right)\right) \omega_{3}\right) \text {. }
$$

Here, $V(\mu)$ denotes the irreducible $\mathfrak{g}$-module of highest weight $\mu \in P^{+}$. If $\mathfrak{g}$ is of type $D_{n}$ with $n \geq 5$ and $\lambda=m_{1} \omega_{1}+m_{2} \omega_{2}+m_{3} \omega_{3}+m_{n-1} \omega_{n-1}+m_{n} \omega_{n}$, the $\mathfrak{g}$-structure of $M(\lambda)$ is given by (1) as well (in this case $a_{i}=1$ for all $i$ ). If $n=4$ and $\lambda \in P^{+}$, then $M(\lambda) \cong \bigoplus_{r=0}^{m_{2}} V\left(\lambda-r \omega_{2}\right)$ as a $\mathfrak{g}$-module.

On the other hand, by regarding the classical limit of a minimal affinization $V_{q}(\lambda)$ as a $\mathfrak{g}[t]$-module and then shifting the associated spectral parameter to zero, we obtain modules $L(\lambda)$, which we call the restricted limit of $V_{q}(\lambda)$. Let $\lambda$ be the maximal weight of $V_{q}(\lambda)$. We prove $T(\lambda)$ is a quotient of $L(\lambda)$ in Proposition 3.21. Moreover, for orthogonal $\mathfrak{g}$, we prove in Proposition 3.22 that $L(\lambda)$ is a quotient of $M(\lambda)$ if $\lambda$ is supported in a connected subdiagram of type $A$ if $\mathfrak{g}$ is of type $D$. Therefore, if indeed $M(\lambda)$ is isomorphic to $T(\lambda)$ as conjectured, it would follow that they are also isomorphic to $L(\lambda)$ in the above cases. In particular, Equation (1) describes the $U_{q}(\mathfrak{g})$-structure of $V_{q}(\lambda)$ when $\mathfrak{g}$ is orthogonal and $\lambda$ is supported only on the first three nodes of the Dynkin diagram of $\mathfrak{g}$ (and possibly on one of the spin nodes if $\mathfrak{g}$ is of type $D$ ). For $\mathfrak{g}$ of type $B_{2}$, Chari [1995] obtained the same result by working purely in the quantum setting. If $\mathfrak{g}$ is of type $B_{n}$ and the value of $\lambda$ on the coroot associated to the spin node is even, then the $\ell$-character (and hence the character) of $V_{q}(\lambda)$ can be computed using the tableaux expression of the JacobiTrudi determinant; see [Chari and Hernandez 2008, Section 7.6]. We expect that, if the minimal connected subdiagram of the Dynkin diagram of $\mathfrak{g}$ containing the support of $\lambda$ does not contain a subdiagram of type $D_{4}$ (in which case $V_{q}(\lambda)$ has a unique equivalence class of minimal affinizations), Proposition 3.22 remains valid and, hence, that the modules $T(\lambda), M(\lambda)$, and $L(\lambda)$ are isomorphic. We will pursue proofs of these conjectures in a more general setting in a forthcoming publication.

When $V_{q}(\lambda)$ has more than one equivalence class of minimal affinizations, it is certainly not true that $L(\lambda)$ is a quotient of $M(\lambda)$ (in fact, it is the other way around). Chari and Pressley [1996b] proved that if $\lambda$ is supported in the triply connected node of the Dynkin diagram of $\mathfrak{g}$, then there are exactly three equivalence classes of minimal affinizations. We define $\mathfrak{g}[t]$-modules $M_{k}(\lambda)$ for $k=1,2,3$ and prove that $L(\lambda)$ is a quotient of $M_{k}(\lambda)$ for exactly one value of $k$. Naturally, we expect that $L(\lambda)$ is isomorphic to the appropriate $M_{k}(\lambda)$. We prove that this so if $\mathfrak{g}$ is of type $D_{4}$ and obtain the character of $M_{k}(\lambda)$ in this case. Namely, let $\lambda=m_{1} \omega_{1}+m_{2} \omega_{2}+m_{3} \omega_{3}+m_{4} \omega_{4}$, where the triply connected node is labeled by 4 , suppose $\{i, j, k\}=\{1,2,3\}$, and let

$$
\mathscr{A}_{k}=\left\{\boldsymbol{r} \in \mathbb{Z}_{\geq 0}^{3}: r_{1} \leq m_{k}, r_{1}+r_{2} \leq \min \left\{m_{i}, m_{j}\right\}, r_{3} \leq m_{4}\right\} .
$$


Then we have an isomorphism of $\mathfrak{g}$-modules:

$$
M_{k}(\lambda) \cong \bigoplus_{r \in A_{k}} V\left(\lambda-\left(r_{1}-r_{2}\right) \omega_{k}-\left(r_{1}+r_{2}\right)\left(\omega_{i}+\omega_{j}\right)-\left(r_{3}-r_{1}\right) \omega_{4}\right) .
$$

If $\lambda$ is not supported in the triply connected node, Chari and Pressley proved in [1996a, Theorem 2.2] that the number of equivalence classes of minimal affinizations of $V_{q}(\lambda)$ grows as $\lambda$ "grows". Although we do not have a general conjecture in this case yet, the definition of $M_{k}(\lambda)$ makes sense in this case as well, and its character is computed in the same way as in the previous case. Moreover, the same proof we applied to the previous case in type $D_{4}$ also proves that, if $\lambda$ satisfies the conditions $(\mathrm{a})_{i, j}$ or $(\mathrm{b})_{i, j}$ of [1996a, Theorem 2.2], then $L(\lambda)$ is isomorphic to $M_{k}(\lambda)$ for the appropriate value of $k$ and its character is given by Equation (2). In particular, this proves the conjecture of [1996a] saying that the modules $V_{q}(\lambda)$ with $\lambda$ satisfying conditions (a) $)_{i, j}$ of [1996a, Theorem 2.2] are equivalent to those with $\lambda$ satisfying conditions (b) $)_{i, j}$ of that theorem.

The techniques used to prove Propositions 3.21 and 3.22 (and their analogues in the case of multiple equivalence classes of minimal affinizations) require the results of [Chari 2002]. For the proof of Proposition 3.22, we also use partial information on $\ell$-characters by combining the Frenkel-Mukhin algorithm with results proved in [CM 2005; Frenkel and Mukhin 2001; Hernandez 2007].

The paper is organized as follows. In Sections 1 and 2, we review some structural results of the algebras $\mathfrak{g}$ and $\tilde{\mathfrak{g}}$ and their quantum counterparts, as well as some basic results of the finite-dimensional representation theory of these algebras. In Section 3, after reviewing the partial classification of minimal affinizations, we define the modules $M(\lambda), T(\lambda)$, and $L(\lambda)$, and state our main results and conjectures regarding them. The proofs are given in Sections 4 and 5. The case of multiple equivalence classes of minimal affinizations is treated in Sections 5.11 and 5.18.

\section{Quantum and classical loop algebras}

Throughout the paper, let $\mathbb{C}, \mathbb{R}, \mathbb{Z}, \mathbb{Z}_{\geq m}$ denote the sets of complex numbers, reals, integers, and integers no less than $m$, respectively. Given a ring $\mathbb{A}$, the underlying multiplicative group of units is denoted by $\mathbb{A}^{\times}$. The dual of a vector space $V$ is denoted by $V^{*}$. The symbol $\cong$ means "isomorphic to".

1.1. Classical algebras. Let $I=\{1, \ldots, n\}$ be the set of vertices of a finite-type connected Dynkin diagram labeled as in [Humphreys 1972], and let $\mathfrak{g}$ be the associated simple Lie algebra over $\mathbb{C}$ with a fixed Cartan subalgebra $\mathfrak{h}$. Fix a set of positive roots $R^{+}$and let

$$
\mathfrak{n}^{ \pm}=\bigoplus_{\alpha \in R^{+}} \mathfrak{g}_{ \pm \alpha}, \quad \text { where } \mathfrak{g}_{ \pm \alpha}=\{x \in \mathfrak{g}:[h, x]= \pm \alpha(h) x, \forall h \in \mathfrak{h}\} .
$$


The simple roots will be denoted by $\alpha_{i}$, the fundamental weights by $\omega_{i}$, while $Q, P, Q^{+}, P^{+}$will denote the root and weight lattices with corresponding positive cones, respectively. Let also $h_{i} \in \mathfrak{h}$ be the coroot associated to $\alpha_{i}$ for $i \in I$. We equip $\mathfrak{h}^{*}$ with the partial order $\lambda \leq \mu$ if and only if $\mu-\lambda \in Q^{+}$. We denote by $\mathscr{W}$ the Weyl group of $\mathfrak{g}$ and let $w_{0}$ be the longest element of $\mathcal{W}$. Given $\lambda \in P$, set

$$
\lambda^{*}=-w_{0} \lambda \text {. }
$$

Recall that if $\lambda \in P^{+}$, then $\lambda^{*} \in P^{+}$as well. Let $C=\left(c_{i j}\right)_{i, j \in I}$ be the Cartan matrix of $\mathfrak{g}$, that is, $c_{i j}=\alpha_{j}\left(h_{i}\right)$, and let $D=\operatorname{diag}\left(d_{i}: i \in I\right)$ be such that the numbers $d_{i}$ are coprime positive integers and $D C$ is symmetric.

The subalgebras $\mathfrak{g}_{ \pm \alpha}$ for $\alpha \in R^{+}$are one-dimensional, and $\left[\mathfrak{g}_{ \pm \alpha}, \mathfrak{g}_{ \pm \beta}\right]=\mathfrak{g}_{ \pm \alpha \pm \beta}$ for every $\alpha, \beta \in R^{+}$. We denote by $x_{\alpha}^{ \pm}$any generator of $\mathfrak{g}_{ \pm \alpha}$. In particular, if $\alpha+\beta \in R^{+}$, then $\left[x_{\alpha}^{ \pm}, x_{\beta}^{ \pm}\right]$is a nonzero generator of $\mathfrak{g}_{ \pm \alpha \pm \beta}$, and we simply write $\left[x_{\alpha}^{ \pm}, x_{\beta}^{ \pm}\right]=x_{\alpha+\beta}^{ \pm}$. For each subset $J$ of $I$, let $\mathfrak{g}_{J}$ be the Lie subalgebra of $\mathfrak{g}$ generated by $x_{\alpha_{j}}^{ \pm}$for $j \in J$, and define $\mathfrak{n}_{J}^{ \pm}$and $\mathfrak{h}_{J}$ in the obvious way. Let also $Q_{J}$ be the subgroup of $Q$ generated by $\alpha_{j}$ for $j \in J$, and let $R_{J}^{+}=R^{+} \cap Q_{J}$. Given $\lambda \in P$, let $\lambda_{J}$ be the restriction of $\lambda$ to $\mathfrak{h}_{J}^{*}$, and let $\lambda^{J} \in P$ be such that $\lambda^{J}\left(h_{j}\right)=\lambda\left(h_{j}\right)$ if $j \in J$ and $\lambda^{J}\left(h_{j}\right)=0$ otherwise. By abuse of language, we will refer to any subset $J$ of $I$ as a subdiagram of the Dynkin diagram of $\mathfrak{g}$. The support of $\mu \in P$ is defined to be the subdiagram $\operatorname{supp}(\mu) \subseteq I$ given by $\operatorname{supp}(\mu)=\left\{i \in I: \mu\left(h_{i}\right) \neq 0\right\}$. Let also $\overline{\operatorname{supp}}(\mu)$ be the minimal connected subdiagram of $I$ containing $\operatorname{supp}(\mu)$.

If $\mathfrak{a}$ is a Lie algebra over $\mathbb{C}$, define its loop algebra to be $\tilde{\mathfrak{a}}=\mathfrak{a} \otimes_{\mathbb{C}} \mathbb{C}\left[t, t^{-1}\right]$ with bracket given by $\left[x \otimes t^{r}, y \otimes t^{s}\right]=[x, y] \otimes t^{r+s}$. Clearly $\mathfrak{a} \otimes 1$ is a subalgebra of $\tilde{\mathfrak{a}}$ isomorphic to $\mathfrak{a}$ and, by abuse of notation, we will continue denoting its elements by $x$ instead of $x \otimes 1$. We also consider the current algebra $\mathfrak{a}[t]$, the subalgebra of $\tilde{\mathfrak{a}}$ given by $\mathfrak{a}[t]=\mathfrak{a} \otimes \mathbb{C}[t]$. Then $\tilde{\mathfrak{g}}=\tilde{\mathfrak{n}}^{-} \oplus \tilde{\mathfrak{h}} \oplus \tilde{\mathfrak{n}}^{+}$and $\tilde{\mathfrak{h}}$ is an abelian subalgebra and similarly for $\mathfrak{g}[t]$. The elements $x_{\alpha}^{ \pm} \otimes t^{r}$ and $h_{i} \otimes t^{r}$ will be denoted by $x_{\alpha, r}^{ \pm}$ and $h_{\alpha_{i}, r}$, respectively. Diagram subalgebras $\tilde{\mathfrak{g}}_{J}$ are defined in the obvious way.

Let $U(\mathfrak{a})$ denote the universal enveloping algebra of a Lie algebra $\mathfrak{a}$. Then $U(\mathfrak{a})$ is a subalgebra of $U(\tilde{\mathfrak{a}})$ and multiplication establishes isomorphisms of vector spaces

$$
U(\mathfrak{g}) \cong U\left(\mathfrak{n}^{-}\right) \otimes U(\mathfrak{h}) \otimes U\left(\mathfrak{n}^{+}\right) \quad \text { and } \quad U(\tilde{\mathfrak{g}}) \cong U\left(\tilde{\mathfrak{n}}^{-}\right) \otimes U(\tilde{\mathfrak{h}}) \otimes U\left(\tilde{\mathfrak{n}}^{+}\right) .
$$

We can uniquely extend the assignments

$\triangle: \mathfrak{a} \rightarrow U(\mathfrak{a}) \otimes U(\mathfrak{a}), x \mapsto x \otimes 1+1 \otimes x, \quad S: \mathfrak{a} \rightarrow \mathfrak{a}, x \mapsto-x, \quad \epsilon: \mathfrak{a} \rightarrow \mathbb{C}, x \mapsto 0$

so that $U(\mathfrak{a})$ becomes a Hopf algebra with comultiplication $\triangle$, antipode $S$, and counit $\epsilon$.

Given $a \in \mathbb{C}$, let $\tau_{a}$ be the Lie algebra automorphism of $\mathfrak{a}[t]$ defined so that $\tau_{a}(x \otimes f(t))=x \otimes f(t-a)$ for every $x \in \mathfrak{a}$ and every $f(t) \in \mathbb{C}[t]$. If $a \neq 0$, let 
$\mathrm{ev}_{a}: \tilde{\mathfrak{a}} \rightarrow \mathfrak{a}$ be the evaluation map $x \otimes f(t) \mapsto f(a) x$. We also denote by $\tau_{a}$ and $\mathrm{ev}_{a}$ the induced maps $U(\mathfrak{a}[t]) \rightarrow U(\mathfrak{a}[t])$ and $U(\tilde{\mathfrak{a}}) \rightarrow U(\mathfrak{a})$, respectively.

For each $i \in I$ and $r \in \mathbb{Z}$, define elements $\Lambda_{i, r} \in U(\tilde{\mathfrak{h}})$ by the following equality of formal power series in the variable $u$ :

$$
\sum_{r=0}^{\infty} \Lambda_{i, \pm r} u^{r}=\exp \left(-\sum_{s=1}^{\infty} \frac{h_{\alpha_{i}, \pm s}}{s} u^{s}\right)
$$

1.2. Quantum algebras. Let $\mathbb{C}(q)$ be the ring of rational functions on an indeterminate $q$, and let $\mathbb{A}=\mathbb{C}\left[q, q^{-1}\right]$. Given $p=q^{k}$ for some $k \in \mathbb{Z} \backslash\{0\}$, define $[m]_{p}=\frac{p^{m}-p^{-m}}{p-p^{-1}}, \quad[m]_{p} !=[m]_{p}[m-1]_{p} \ldots[2]_{p}[1]_{p},\left[\begin{array}{c}m \\ r\end{array}\right]_{p}=\frac{[m]_{p} !}{[r]_{p} ![m-r]_{p} !}$, for $r, m \in \mathbb{Z}_{\geq 0}$ with $m \geq r$. Notice that $\left[\begin{array}{c}m \\ r\end{array}\right]_{p} \in \mathbb{A}$.

Set $q_{i}=q^{d_{i}}$. The quantum loop algebra $U_{q}(\tilde{\mathfrak{g}})$ of $\mathfrak{g}$ is the algebra with generators $x_{i, r}^{ \pm}$for $i \in I, r \in \mathbb{Z}, \quad k_{i}^{ \pm 1}$ for $i \in I, \quad h_{i, r}$ for $i \in I, r \in \mathbb{Z} \backslash\{0\}$, and the defining relations

$$
\begin{aligned}
& k_{i} k_{i}^{-1}=k_{i}^{-1} k_{i}=1, \quad k_{i} k_{j}=k_{j} k_{i}, \\
& k_{i} h_{j, r}=h_{j, r} k_{i}, \quad k_{i} x_{j, r}^{ \pm} k_{i}^{-1}=q_{i}^{ \pm c_{i j}} x_{j, r}^{ \pm}, \\
& {\left[h_{i, r}, h_{j, s}\right] }=0, \quad\left[h_{i, r}, x_{j, s}^{ \pm}\right]= \pm \frac{1}{r}\left[r c_{i j}\right]_{q^{i}} x_{j, r+s}^{ \pm}, \\
& x_{i, r+1}^{ \pm} x_{j, s}^{ \pm}-q_{i}^{ \pm c_{i j}} x_{j, s}^{ \pm} x_{i, r+1}^{ \pm}=q_{i}^{ \pm c_{i j}} x_{i, r}^{ \pm} x_{j, s+1}^{ \pm}-x_{j, s+1}^{ \pm} x_{i, r}^{ \pm}, \\
& {\left[x_{i, r}^{+}, x_{j, s}^{-}\right] }=\delta_{i, j} \frac{\psi_{i, r+s}^{+}-\psi_{i, r+s}^{-}}{q_{i}-q_{i}^{-1}}, \\
& \sum_{\sigma \in S_{m}} \sum_{k=0}^{m}(-1)^{k}\left[\begin{array}{c}
m \\
k
\end{array}\right]_{q_{i}} x_{i, r_{\sigma(1)}}^{ \pm} \ldots x_{i, r_{\sigma(k)}}^{ \pm} x_{j, s}^{ \pm} x_{i, r_{\sigma(k+1)}}^{ \pm} \ldots x_{i, r_{\sigma(m)}}^{ \pm}=0 \quad \text { if } i \neq j,
\end{aligned}
$$

for all sequences of integers $r_{1}, \ldots, r_{m}$, where $m=1-c_{i j}, S_{m}$ is the symmetric group on $m$ letters, and the $\psi_{i, r}^{ \pm}$are determined by equating powers of $u$ in the formal power series

$$
\Psi_{i}^{ \pm}(u)=\sum_{r=0}^{\infty} \psi_{i, \pm r}^{ \pm} u^{r}=k_{i}^{ \pm 1} \exp \left( \pm\left(q_{i}-q_{i}^{-1}\right) \sum_{s=1}^{\infty} h_{i, \pm s} u^{s}\right) .
$$

Denote by $U_{q}\left(\tilde{\mathfrak{n}}^{ \pm}\right)$and $U_{q}(\tilde{\mathfrak{h}})$ the subalgebras of $U_{q}(\tilde{\mathfrak{g}})$ generated by $\left\{x_{i, r}^{ \pm}\right\}$and $\left\{k_{i}^{ \pm 1}, h_{i, s}\right\}$, respectively. Let $U_{q}(\mathfrak{g})$ be the subalgebra generated by $x_{i}^{ \pm}:=x_{i, 0}^{ \pm}$ and $k_{i}^{ \pm 1}$ for $i \in I$, and define $U_{q}\left(\mathfrak{n}^{ \pm}\right)$and $U_{q}(\mathfrak{h})$ in the obvious way. $U_{q}(\mathfrak{g})$ is a 
subalgebra of $U_{q}(\tilde{\mathfrak{g}})$, and multiplication establishes isomorphisms

$$
U_{q}(\mathfrak{g}) \cong U_{q}\left(\mathfrak{n}^{-}\right) \otimes U_{q}(\mathfrak{h}) \otimes U_{q}\left(\mathfrak{n}^{+}\right) \quad \text { and } \quad U_{q}(\tilde{\mathfrak{g}}) \cong U_{q}\left(\tilde{\mathfrak{n}}^{-}\right) \otimes U_{q}(\tilde{\mathfrak{h}}) \otimes U_{q}\left(\tilde{\mathfrak{n}}^{+}\right)
$$

of $\mathbb{C}(q)$-vectors spaces.

Let $J \subseteq I$ and consider the subalgebra $U_{q}\left(\tilde{\mathfrak{g}}_{J}\right)$ generated by $k_{j}^{ \pm 1}, h_{j, r}, x_{j, s}^{ \pm}$for all $j \in J, r, s \in \mathbb{Z}$ with $r \neq 0$. If $J=\{j\}$, the algebra $U_{q}\left(\tilde{\mathfrak{g}}_{j}\right):=U_{q}\left(\tilde{\mathfrak{g}}_{J}\right)$ is isomorphic to $U_{q_{j}}\left(\tilde{\mathfrak{s}}_{2}\right)$. Similarly we define the subalgebra $U_{q}\left(\mathfrak{g}_{J}\right)$, and so on.

For $i \in I, r \in \mathbb{Z}$, and $k \in \mathbb{Z}_{\geq 0}$, define $\left(x_{i, r}^{ \pm}\right)^{(k)}=\left(x_{i, r}^{ \pm}\right)^{k} /[k]_{q_{i}} !$. Define also elements $\Lambda_{i, r}$ for $i \in I$ and $r \in \mathbb{Z}$ by

$$
\sum_{r=0}^{\infty} \Lambda_{i, \pm r} u^{r}=\exp \left(-\sum_{s=1}^{\infty} \frac{h_{i, \pm s}}{[s]_{q_{i}}} u^{s}\right) .
$$

Note that

$$
\Psi_{i}^{ \pm}(u)=k_{i}^{ \pm 1} \frac{\Lambda_{i}^{ \pm}\left(q_{i}^{\mp 1} u\right)}{\Lambda_{i}^{ \pm}\left(q_{i}^{ \pm 1} u\right)},
$$

where the division is that of formal power series in $u$. Although we are denoting the elements $\Lambda_{i, r}$ above by the same symbol as their classical counterparts, this will not create confusion as it will be clear from the context.

Let $U_{\mathbb{A}}(\tilde{\mathfrak{g}})$ be the $\mathbb{A}$-subalgebra of $U_{q}(\tilde{\mathfrak{g}})$ generated by the elements $\left(x_{i, r}^{ \pm}\right)^{(k)}$ and $k_{i}^{ \pm 1}$ for $i \in I, \quad r \in \mathbb{Z}$, and $k \in \mathbb{Z}_{\geq 0}$. Define $U_{\mathbb{A}}(\mathfrak{g})$ similarly and notice that $U_{\mathrm{A}}(\mathfrak{g})=U_{\mathrm{A}}(\tilde{\mathfrak{g}}) \cap U_{q}(\mathfrak{g})$. For the proof of the next proposition see [Chari 2001, Lemma 2.1] and the locally cited references.

Proposition 1.3. $U_{q}(\tilde{\mathfrak{g}})=\mathbb{C}(q) \otimes_{\mathbb{A}} U_{\mathbb{A}}(\tilde{\mathfrak{g}})$ and $U_{q}(\mathfrak{g})=\mathbb{C}(q) \otimes_{\mathbb{A}} U_{\mathbb{A}}(\mathfrak{g})$.

Regard $\mathbb{C}$ as an $\mathbb{A}$-module by letting $q$ act as 1 , and set

$$
\overline{U_{q}(\tilde{\mathfrak{g}})}=\mathbb{C} \otimes_{\mathbb{A}} U_{\mathbb{A}}(\tilde{\mathfrak{g}}) \text { and } \overline{U_{q}(\mathfrak{g})}=\mathbb{C} \otimes_{\mathbb{A}} U_{\mathbb{A}}(\mathfrak{g}) .
$$

Denote by $\bar{\eta}$ the image of $\eta \in U_{\mathrm{A}}(\tilde{\mathfrak{g}})$ in $\overline{U_{q}(\tilde{\mathfrak{g}})}$. The proof of the next proposition can be found in [CP 1994a, Proposition 9.2.3] and [Lusztig 1993].

Proposition 1.4. $U(\tilde{\mathfrak{g}})$ is isomorphic to the quotient of $\overline{U_{q}(\tilde{\mathfrak{g}})}$ by the ideal generated by $\bar{k}_{i}-1$. In particular, the category of $\overline{U_{q}(\tilde{\mathfrak{g}})}$-modules on which the $k_{i}$ act as the identity operator for all $i \in I$ is equivalent to the category of all $\tilde{\mathfrak{g}}$-modules.

The algebra $U_{q}(\tilde{\mathfrak{g}})$ is a Hopf algebra and induces a Hopf algebra structure (over $\mathbb{A}$ ) on $U_{\mathbb{A}}(\tilde{\mathfrak{g}})$; see [CP 1994a; Lusztig 1993]. The induced Hopf algebra structure on $U(\tilde{\mathfrak{g}})$ coincides with the usual one. On $U_{q}(\mathfrak{g})$ we have, for all $i \in I$,

$$
\Delta\left(x_{i}^{+}\right)=x_{i}^{+} \otimes 1+k_{i} \otimes x_{i}^{+}, \quad \Delta\left(x_{i}^{-}\right)=x_{i}^{-} \otimes k_{i}^{-1}+1 \otimes x_{i}^{-}, \quad \Delta\left(k_{i}\right)=k_{i} \otimes k_{i} .
$$


Lemma 1.5. Suppose $x=\left[x_{i_{1}}^{-},\left[x_{i_{2}}^{-}, \cdots\left[x_{i_{l-1}}^{-}, x_{i_{l}}^{-}\right] \cdots\right]\right]$. Then $X \in U_{\mathbb{A}}\left(\mathfrak{n}^{-}\right)$and

$$
\Delta(x) \in x \otimes\left(\prod_{j=1}^{l} k_{i_{j}}^{-1}\right)+1 \otimes x+f(q) y
$$

for some $y \in U_{\mathbb{A}}(\mathfrak{g}) \otimes U_{\mathbb{A}}(\mathfrak{g})$ and some $f(q) \in \mathbb{A}$ such that $f(1)=0$.

Proof. When $l=1$, this is immediate from (1-6). A straightforward induction on $l$ using the relations $k_{i} x_{j}^{-}=q_{i}^{-c_{i, j}} x_{j}^{-} k_{i}$ completes the proof.

An expression for the comultiplication $\Delta$ of $U_{q}(\tilde{\mathfrak{g}})$ in terms of the generators $x_{i, r}^{ \pm}, h_{i}, r, k_{i}^{ \pm 1}$ is not known. The following partial information will suffice for our purposes. Let $X^{ \pm}$be the subspace of $U_{\mathbb{A}}\left(\tilde{\mathfrak{n}}^{ \pm}\right)$spanned by $\left\{x_{j, r}^{ \pm}: j \in I, r \in \mathbb{Z}\right\}$.

Lemma 1.6. $\Delta\left(x_{i, 1}^{-}\right)=x_{i, 1}^{-} \otimes k_{i}+1 \otimes x_{i, 1}^{-}+x$ for some $x \in U_{\mathbb{A}}(\mathfrak{g}) \otimes U_{\mathbb{A}}(\mathfrak{g})$ such that $\bar{x}=0$.

Proof. It was proved in [Beck 1994; Beck et al. 1999; Damiani 1998] - see also [CP 1997, Lemma 7.5] - that $\Delta\left(x_{i, 1}^{-}\right)=x_{i, 1}^{-} \otimes k_{i}+1 \otimes x_{i, 1}^{-}+x$, where $x \in U_{\mathbb{A}}(\tilde{\mathfrak{g}}) X^{-} \otimes U_{\mathbb{A}}(\tilde{\mathfrak{g}}) X^{+}$. Since the image $\overline{\Delta\left(x_{i, 1}^{-}\right)}$of $\Delta\left(x_{i, 1}^{-}\right)$in $U(\tilde{\mathfrak{g}}) \otimes U(\tilde{\mathfrak{g}})$ is $x_{\alpha_{i}, 1}^{-} \otimes 1+1 \otimes x_{\alpha_{i}, 1}^{-}$, the image of $x$ in $U(\tilde{\mathfrak{g}}) \otimes U(\tilde{\mathfrak{g}})$ must be zero.

The following was also proved in [Beck 1994; Beck et al. 1999; Damiani 1998]. Modulo $U_{q}(\tilde{\mathfrak{g}}) X^{-} \otimes U_{q}(\tilde{\mathfrak{g}}) X^{+}$, we have, for all $r \in \mathbb{Z}_{\geq 1}$,

$$
\Delta\left(h_{i, r}\right)=h_{i, s} \otimes 1+1 \otimes h_{i, r} \quad \text { and } \quad \Delta\left(\Lambda_{i, r}\right)=\sum_{s=0}^{r} \Lambda_{i, r-s} \otimes \Lambda_{i, s}
$$

The following general result on the dual representation of a tensor product of representations of a Hopf algebra is proved, for instance, in [Kassel 1995].

Proposition 1.7. Let $H$ be a Hopf algebra and $V$ and $W$ be finite-dimensional $H$-modules. Then $(V \otimes W)^{*} \cong W^{*} \otimes V^{*}$.

1.8. The $\ell$-weight lattice. Given a field $\mathbb{F}$ consider the multiplicative group $\mathscr{P}_{\mathbb{F}}$ of $n$-tuples of rational functions $\boldsymbol{\mu}=\left(\boldsymbol{\mu}_{1}(u), \ldots, \boldsymbol{\mu}_{n}(u)\right)$ with values in $\mathbb{F}$ such that $\boldsymbol{\mu}_{i}(0)=1$ for all $i \in I$. We shall often think of $\boldsymbol{\mu}_{i}(u)$ as a formal power series in $u$ with coefficients in $\mathbb{F}$. Given $a \in \mathbb{F}^{\times}$and $i \in I$, let $\omega_{i, a}$ be defined by

$$
\left(\omega_{i, a}\right)_{j}(u)=1-\delta_{i, j} a u .
$$

Clearly, if $\mathbb{F}$ is algebraically closed, $\mathscr{P}_{\mathbb{F}}$ is the free abelian group generated by these elements, which are called fundamental $\ell$-weights. It is also convenient to introduce elements $\boldsymbol{\omega}_{\lambda, a}$ for $\lambda \in P$ and $a \in \mathbb{C}(q)$ defined by

$$
\boldsymbol{\omega}_{\lambda, a}=\prod_{i \in I}\left(\boldsymbol{\omega}_{i, a}\right)^{\lambda\left(h_{i}\right)} .
$$


If $\mathbb{F}$ is algebraically closed, introduce the group homomorphism (weight map) wt $: \mathscr{P}_{\mathbb{F}} \rightarrow P$ by setting $\operatorname{wt}\left(\omega_{i, a}\right)=\omega_{i}$, where $\omega_{i}$ is the $i$-th fundamental weight of $\mathfrak{g}$. Otherwise, let $\mathbb{K}$ be an algebraically closed extension of $\mathbb{E}$, so that $\mathscr{P}_{\mathbb{F}}$ can be regarded as a subgroup of $\mathscr{P}_{\mathbb{K}}$, and define the weight map on $\mathscr{P}_{\mathbb{F}}$ by restricting the one on $\mathscr{P}_{\mathbb{K}}$ (this clearly does not depend on the choice of $\mathbb{K}$ ). Define the $\ell$-weight lattice of $U_{q}(\tilde{\mathfrak{g}})$ to be $\mathscr{P}_{q}:=\mathscr{P}_{\mathbb{C}(q)}$. The submonoid $\mathscr{P}_{q}^{+}$of $\mathscr{P}_{q}$ consisting of $n$-tuples of polynomials is called the set of dominant $\ell$-weights of $U_{q}(\tilde{\mathfrak{g}})$.

Given $\lambda \in \mathscr{P}_{q}^{+}$with $\lambda_{i}(u)=\prod_{j}\left(1-a_{i, j} u\right)$, where $a_{i, j}$ belongs to some algebraic closure of $\mathbb{C}(q)$, let $\lambda^{-} \in \mathscr{P}_{q}^{+}$be defined by $\lambda_{i}^{-}(u)=\prod_{j}\left(1-a_{i, j}^{-1} u\right)$. We will also use the notation $\lambda^{+}=\lambda$. Two elements $\lambda, \mu$ of $\mathscr{P}_{q}^{+}$are said to be relatively prime if $\lambda_{i}(u)$ is relatively prime to $\boldsymbol{\mu}_{j}(u)$ in $\mathbb{C}(q)[u]$ for all $i, j \in I$. Every $\boldsymbol{v} \in \mathscr{P}_{q}$ can be uniquely written in the form

$$
\boldsymbol{v}=\lambda \boldsymbol{\mu}^{-1} \text { with } \lambda, \boldsymbol{\mu} \in \mathscr{P}_{q}^{+} \text {relatively prime. }
$$

Given this $\boldsymbol{v}=\lambda \boldsymbol{\mu}^{-1}$, define a $\mathbb{C}(q)$-algebra homomorphism $\boldsymbol{\Psi}_{\boldsymbol{v}}: U_{q}(\tilde{\mathfrak{h}}) \rightarrow \mathbb{C}(q)$ by setting

$$
\boldsymbol{\Psi}_{\boldsymbol{v}}\left(k_{i}^{ \pm 1}\right)=q_{i}^{ \pm \operatorname{wt}(\boldsymbol{v})\left(h_{i}\right)} \quad \text { and } \quad \sum_{r \geq 0} \boldsymbol{\Psi}_{\boldsymbol{v}}\left(\Lambda_{i, \pm r}\right) u^{r}=\frac{\left(\lambda^{ \pm}\right)_{i}(u)}{\left(\boldsymbol{\mu}^{ \pm}\right)_{i}(u)},
$$

where the division is that of formal power series in $u$. The next proposition is easy.

Proposition 1.9. The map $\boldsymbol{\Psi}: \mathscr{P}_{q} \rightarrow\left(U_{q}(\tilde{\mathfrak{h}})\right)^{*}$ given by $\boldsymbol{v} \mapsto \boldsymbol{\Psi}_{\boldsymbol{v}}$ is injective.

Define the $\ell$-weight lattice $\mathscr{P}$ of $\tilde{\mathfrak{g}}$ to be the subgroup of $\mathscr{P}_{q}$ generated by $\omega_{i, a}$ for all $i \in I$ and all $a \in \mathbb{C}^{\times}$or, equivalently, $\mathscr{P}=\mathscr{P}_{\mathbb{C}}$. Observe that every element $\lambda \in \mathscr{P}$ can be uniquely decomposed as

$$
\lambda=\prod_{j} \omega_{\lambda_{j}, a_{j}} \quad \text { for some } \lambda_{j} \in P \text { and } a_{i} \neq a_{j} \in \mathbb{C} .
$$

Set also $\mathscr{P}^{+}=\mathscr{P} \cap \mathscr{P}_{q}^{+}$.

From now on we will identify $\mathscr{P}_{q}$ with its image in $\left(U_{q}(\tilde{\mathfrak{h}})\right)^{*}$ under $\boldsymbol{\Psi}$. Similarly, $\mathscr{P}$ will be identified with a subset of $U(\tilde{\mathfrak{h}})^{*}$ via the homomorphism $\Psi_{v}: U(\tilde{\mathfrak{h}}) \rightarrow \mathbb{C}$ determined by

$$
\boldsymbol{\Psi}_{\boldsymbol{v}}\left(h_{i}\right)=\operatorname{wt}(\boldsymbol{v})\left(h_{i}\right) \quad \text { and } \quad \sum_{r \geq 0} \boldsymbol{\Psi}_{\boldsymbol{v}}\left(\Lambda_{i, \pm r}\right) u^{r}=\frac{\left(\lambda^{ \pm}\right)_{i}(u)}{\left(\boldsymbol{\mu}^{ \pm}\right)_{i}(u)} .
$$

It will be convenient to introduce the following notation. Given $i \in I, a \in \mathbb{C}(q)^{\times}$, and $r \in \mathbb{Z}_{\geq 0}$, define

$$
\boldsymbol{\omega}_{i, a, r}=\prod_{j=0}^{r-1} \boldsymbol{\omega}_{i, a q_{i}^{r-1-2 j}}
$$


Define also the polynomial

$$
f_{i, a, r}(u)=\prod_{j=0}^{r-1}\left(1-a q_{i}^{r-1-2 j} u\right) .
$$

Observe that given $f(u) \in \mathbb{C}(q)[u]$ having all its roots in $\mathbb{C}(q)$ and such that $f(1)=0$, there exist unique $m \in \mathbb{Z}_{\geq 0}, a_{1}, \ldots, a_{m} \in \mathbb{C}(q)^{\times}$, and $r_{1}, \ldots, r_{m} \in \mathbb{Z}_{\geq 1}$ such that

(1-15) $f(u)=\prod_{k=1}^{m} f_{i, a_{k}, r_{k}}(u) \quad$ with $\frac{a_{l}}{a_{j}} \neq q^{ \pm\left(r_{l}+r_{j}-2 p\right)}$ for $0 \leq p<\min \left\{r_{l}, r_{j}\right\}$.

In particular, given $\lambda \in \mathscr{P}_{q}^{+}$such that $\lambda_{i}(u)$ splits in $\mathbb{C}(q)[u]$ for all $i \in I$, there exist unique $m_{i} \in Z_{\geq 0}, a_{i, k} \in \mathbb{C}(q)^{\times}$, and $r_{i, k} \in \mathbb{Z}_{\geq 1}$ such that

$$
\begin{aligned}
\lambda=\prod_{i \in I} \prod_{k=1}^{m_{i}} \omega_{i, a_{i, k}, r_{i, k},} & \\
\text { with } & \frac{a_{i, j}}{a_{i, l}} \neq q_{i}^{ \pm\left(r_{i, j}+r_{i, l}-2 p\right)} \text { and } \sum_{k=1}^{m_{i}} r_{i, k}=\operatorname{wt}(\lambda)\left(h_{i}\right) \\
& \quad \text { for all } i \in I, j \neq l, \text { and } 0 \leq p<\min \left\{r_{i, j}, r_{i, l}\right\} .
\end{aligned}
$$

If $J \subseteq I$ and $\lambda \in \mathscr{P}_{q}$, let $\lambda_{J}$ be the associated $J$-tuple of rational functions. Note that if $\lambda_{j}(u) \in \mathbb{C}\left(q_{j}\right)(u)$ for all $j \in J$, then $\lambda_{J}$ can be regarded as an element of the $\ell$-weight lattice of $U_{q}\left(\tilde{\mathfrak{g}}_{J}\right)$. Let also $\lambda^{J} \in \mathscr{P}_{q}$ be such that $\left(\lambda^{J}\right)_{j}(u)=\lambda_{j}(u)$ for every $j \in J$ and $\left(\lambda^{J}\right)_{j}(u)=1$ otherwise.

Recall that $w_{0}$ defines a Dynkin diagram automorphism such that $w_{0} \cdot i=j$ if and only if $w_{0} \omega_{i}=-\omega_{j}$ for $i, j \in I$. Given $\lambda \in \mathscr{P}_{q}^{+}$, let $\lambda^{*} \in \mathscr{P}_{q}^{+}$be the element defined by

$$
\left(\lambda^{*}\right)_{i}(u)=\lambda_{w_{0} \cdot i}\left(q^{r^{\vee} h^{\vee}} u\right)
$$

where $h^{\vee}$ is the dual Coxeter number of $\mathfrak{g}$ and $r^{\vee}=\max \left\{c_{i j} c_{j i}: i, j \in I, i \neq j\right\}$ is the lacing number of $\mathfrak{g}$. Define also the element ${ }^{*} \lambda$ by requiring $\left({ }^{*} \lambda\right)^{ \pm}=\left(\lambda^{*}\right)^{\mp}$.

Given $i \in I$ and $a \in \mathbb{C}(q)^{\times}$, define the simple $\ell$-root $\boldsymbol{\alpha}_{i, a}$ by

$$
\boldsymbol{\alpha}_{i, a}=\left(\boldsymbol{\omega}_{i, a q_{i}, 2}\right)^{-1} \prod_{j \neq i} \omega_{j, a q_{i},-c_{j, i}} .
$$

The subgroup of $\mathscr{P}_{q}$ generated by the simple $\ell$-roots is called the $\ell$-root lattice of $U_{q}(\tilde{\mathfrak{g}})$ and will be denoted by $2_{q}$. Let also $2_{q}^{+}$be the submonoid generated by the simple $\ell$-roots. Clearly $\operatorname{wt}\left(\boldsymbol{\alpha}_{i, a}\right)=\alpha_{i}$. Define a partial order on $\mathscr{P}_{q}$ by $\boldsymbol{\mu} \leq \boldsymbol{\lambda}$ if $\lambda \boldsymbol{\mu}^{-1} \in 2_{q}^{+}$. 


\section{Finite-dimensional representations}

2.1. Simple Lie algebras. We now review some of basic facts of the representation theory of $\mathfrak{g}$ and $U_{q}(\mathfrak{g})$. For details, see for instance [Humphreys 1972] or CP 1994a].

Given a $\mathfrak{g}$-module $V$ and $\mu \in \mathfrak{h}^{*}$, let $V_{\mu}=\{v \in V: h v=\mu(h) v$ for all $h \in \mathfrak{h}\}$. A nonzero vector $v \in V_{\mu}$ is called a weight vector of weight $\mu$. If $v$ is a weight vector such that $\mathfrak{n}^{+} v=0$, then $v$ is called a highest-weight vector. If $V$ is generated by a highest-weight vector of weight $\lambda$, then $V$ is said to be a highest-weight module of highest weight $\lambda$.

We summarize the basic facts about finite-dimensional $\mathfrak{g}$-modules:

Theorem 2.2. Let $V$ be a finite-dimensional $\mathfrak{g}$-module.

(a) $V=\bigoplus_{\mu \in P} V_{\mu}$ and $\operatorname{dim} V_{\mu}=\operatorname{dim} V_{w \mu}$ for all $w \in \mathscr{W}$.

(b) $V$ is completely reducible.

(c) For each $\lambda \in P^{+}$, the $U(\mathfrak{g})$-module $V(\lambda)$ generated by a vector $v$ satisfying

$$
x_{\alpha_{i}}^{+} v=0, \quad h_{i} v=\lambda\left(h_{i}\right) v, \quad\left(x_{\alpha_{i}}^{-}\right)^{\lambda\left(h_{i}\right)+1} v=0 \quad \text { for all } i \in I
$$

is irreducible and finite-dimensional. If $V$ is irreducible, then $V$ is isomorphic to $V(\lambda)$ for some $\lambda \in P^{+}$.

(d) If $\lambda \in P^{+}$and $V \cong V(\lambda)$, then $V_{\mu} \neq 0$ if and only if $w \mu \leq \lambda$ for all $w \in W$. Furthermore, the lowest weight of $V(\lambda)$ is $-\lambda^{*}$. In particular, $V(\lambda)^{*} \cong V\left(\lambda^{*}\right)$.

Lemma 2.3. Let $V$ be a finite-dimensional $\mathfrak{g}$-module, and suppose $l \in \mathbb{Z}_{\geq 1}, v_{k} \in P$, and $v_{k} \in V_{v_{k}}$ for $k=1, \ldots$, l are such that $V=\sum_{k=1}^{l} U\left(\mathfrak{n}^{-}\right) v_{k}$. Fix a decomposition $V=\bigoplus_{j=1}^{m} V_{j}$ where $m \in \mathbb{Z}_{\geq 1}$ and $V_{j} \cong V\left(\mu_{j}\right)$ for some $\mu_{j} \in P^{+}$, and let $\pi_{j}: V \rightarrow V_{j}$ be the associated projection for $j=1, \ldots, m$. Then, there exist distinct $k_{1}, \ldots, k_{m} \in\{1, \ldots, l\}$ such that $v_{k_{j}}=\mu_{j}$ and $\pi_{j}\left(v_{k_{j}}\right) \neq 0$.

Proof. Proceed by induction on $m$. If $m=1$, the lemma is immediate. Otherwise, suppose, without loss of generality, that $\mu_{m}$ is a maximal weight of $V$. In that case, there must exist $k_{m}$ such that $v_{k_{m}}=\mu_{m}$ and $v_{k_{m}}$ generates an irreducible submodule of $V$ isomorphic to $V\left(\mu_{m}\right)$. In particular, there exists $j$ such that $\mu_{j}=\mu_{m}$ and $\pi_{j}\left(v_{k_{m}}\right) \neq 0$. Up to reordering, we can assume $j=m$. The lemma now easily follows from the induction hypothesis applied to $\bar{V}:=V / U(\mathfrak{g}) v_{k_{m}}$ and the induced decomposition $\bar{V}=\bigoplus_{j=1}^{m-1} \bar{V}_{j}$, where $\bar{V}_{j}$ is the image of $V_{j}$ in $\bar{V}$.

Let $\mathbb{Z}[P]$ be the integral group ring over $P$ and denote by $e: P \rightarrow \mathbb{Z}[P], \lambda \mapsto$ $e^{\lambda}$, the inclusion of $P$ in $\mathbb{Z}[P]$, so that $e^{\lambda} e^{\mu}=e^{\lambda+\mu}$. Given a finite-dimensional $\mathfrak{g}$-module $V$, the character of $V$ is defined to be

$$
\operatorname{ch}(V)=\sum_{\mu \in P} \operatorname{dim}\left(V_{\mu}\right) e^{\mu} .
$$


Given a $U_{q}(\mathfrak{g})$-module $V$ and $\mu \in P$, let

$$
V_{\mu}=\left\{v \in V: k_{i} v=q_{i}^{\mu\left(h_{i}\right)} v \text { for all } i \in I\right\} .
$$

A nonzero vector $v \in V_{\mu}$ is called a weight vector of weight $\mu$. If $v$ is a weight vector such that $x_{i}^{+} v=0$ for all $i \in I$, then $v$ is called a highest-weight vector. If $V$ is generated by a highest-weight vector of weight $\lambda$, then $V$ is said to be a highestweight module of highest weight $\lambda$. A $U_{q}(\mathfrak{g})$-module $V$ is said to be a weight module if $V=\bigoplus_{\mu \in P} V_{\mu}$. Denote by $\mathscr{C}_{q}$ be the category of all finite-dimensional weight modules of $U_{q}(\mathfrak{g})$.

Remark. A $U_{q}(\mathfrak{g})$-module $V$ satisfying $V=\bigoplus_{\mu \in P} V_{\mu}$ is usually called a weightmodule of type 1. We shall not discuss what type $\mathbf{1}$ means here. For further details see [CP 1994a] for instance.

The character of an object $V \in \mathscr{C}_{q}$ is defined by (2-1). The following theorem is the quantum analogue of Theorem 2.2.

Theorem 2.4. Let $V \in \mathscr{C}_{q}$.

(a) $\operatorname{dim} V_{\mu}=\operatorname{dim} V_{w \mu}$ for all $w \in \mathcal{W}$.

(b) $V$ is completely reducible.

(c) For each $\lambda \in P^{+}$, the $U(\mathfrak{g})$-module $V_{q}(\lambda)$ generated by a vector $v$ satisfying

$$
x_{i}^{+} v=0, \quad k_{i} v=q_{i}^{\lambda\left(h_{i}\right)} v, \quad\left(x_{i}^{-}\right)^{\lambda\left(h_{i}\right)+1} v=0 \quad \text { for all } i \in I
$$

is irreducible and finite-dimensional. If $V$ is irreducible, then $V$ is isomorphic to $V_{q}(\lambda)$ for some $\lambda \in P^{+}$.

(d) If $\lambda \in P^{+}$and $V \cong V_{q}(\lambda)$, then $\operatorname{ch}(V)=\operatorname{ch}\left(V(\lambda)\right.$ ). In particular, $V_{q}(\lambda)^{*}$ is isomorphic to $V_{q}\left(\lambda^{*}\right)$.

If $J \subseteq I$, we shall denote by $V_{q}\left(\lambda_{J}\right)$ the $U_{q}\left(\mathfrak{g}_{J}\right)$-irreducible module of highest weight $\lambda_{J}$. Similarly $V\left(\lambda_{J}\right)$ denotes the corresponding irreducible $\mathfrak{g}_{J}$-module.

2.5. Loop algebras. Let $V$ be a $U_{q}(\tilde{\mathfrak{g}})$-module. We say that a nonzero vector $v \in V$ is an $\ell$-weight vector if there exists $\lambda \in \mathscr{P}_{q}$ and $k \in \mathbb{Z}_{>0}$ such that $\left(\eta-\Psi_{\lambda}(\eta)\right)^{k} v=0$ for all $\eta \in U_{q}(\tilde{\mathfrak{h}})$. In that case, we call $\lambda$ the $\ell$-weight of $v$. We say $V$ is an $\ell$-weight module if every vector of $V$ is a linear combination of $\ell$-weight vectors. In that case, let $V_{\lambda}$ denote the subspace spanned by all $\ell$-weight vectors of $\ell$-weight $\lambda$. An $\ell$-weight vector $v$ is said to be a highest- $\ell$-weight vector if $\eta v=\boldsymbol{\Psi}_{\lambda}(\eta) v$ for every $\eta \in U_{q}(\tilde{\mathfrak{h}})$ and $x_{i, r}^{+} v=0$ for all $i \in I$ and all $r \in \mathbb{Z}$. We say $V$ is a highest$\ell$-weight module if it is generated by a highest- $\ell$-weight vector. The notion of lowest- $\ell$-weight module is defined similarly. Denote by $\widetilde{\mathscr{C}}_{q}$ the category of all finite-dimensional $\ell$-weight modules of $U_{q}(\tilde{\mathfrak{g}})$. Clearly $\widetilde{\mathscr{C}}_{q}$ is an abelian category. 
Observe that if $V \in \widetilde{\mathscr{C}}_{q}$, then $V \in \mathscr{C}_{q}$ and

$$
V_{\lambda}=\bigoplus_{\lambda: \operatorname{wt}(\lambda)=\lambda} V_{\lambda}
$$

Moreover, if $V$ is a highest- $\ell$-weight module of highest $\ell$-weight $\lambda$, then

$$
\operatorname{dim}\left(V_{\mathrm{wt}(\lambda)}\right)=1 \quad \text { and } \quad V_{\mu} \neq 0 \text { implies } \mu \leq \mathrm{wt}(\lambda) .
$$

Define the concepts of $\ell$-weight vector and so on for $\tilde{\mathfrak{g}}$ in a similar way, and denote by $\widetilde{\mathscr{C}}$ the category of all finite-dimensional $\tilde{\mathfrak{g}}$-modules. The next proposition is easily established using (2-3).

Proposition 2.6. If $V$ is a highest- $\ell$-weight module, then it has a unique proper submodule and hence a unique irreducible quotient.

Definition 2.7. Let $\lambda \in \mathscr{P}_{q}^{+}$and $\lambda=\operatorname{wt}(\lambda)$. The Weyl module $W_{q}(\lambda)$ of highest $\ell$-weight $\lambda$ is the $U_{q}(\tilde{\mathfrak{g}})$-module defined by the quotient of $U_{q}(\tilde{\mathfrak{g}})$ by the left ideal generated by the elements $x_{i, r}^{+},\left(x_{i, r}^{-}\right)^{\lambda\left(h_{i}\right)+1}$, and $\eta-\Psi_{\lambda}(\eta)$ for every $i \in I, r \in \mathbb{Z}$, and $\eta \in U_{q}(\tilde{\mathfrak{h}})$. Denote by $V_{q}(\lambda)$ the irreducible quotient of $W_{q}(\lambda)$. For $\lambda \in \mathscr{P}^{+}$, the Weyl module $W(\lambda)$ of $\tilde{\mathfrak{g}}$ and its irreducible quotient $V(\lambda)$ are defined similarly.

The next theorem was proved in [CP 2001, Lemmas 4.6 and 4.7] for simply laced $\mathfrak{g}$ and in [Chari 2001, Proposition 2.2] for $\mathfrak{g}$ with lacing number $r^{\vee}=2$. For the sake of completeness, a proof for $\mathfrak{g}$ of type $G_{2}$ will appear in [Jakelić and Moura 2009b].

Theorem 2.8. For each $\lambda \in \mathscr{P}_{q}^{+}$, the module $W_{q}(\lambda)$ is the universal finite-dimensional $U_{q}(\tilde{\mathfrak{g}})$-module with highest $\ell$-weight $\lambda$; every simple object of $\widetilde{\mathscr{C}}_{q}$ is highest$\ell$-weight. For every $\lambda \in \mathscr{P}^{+}$, the module $W(\lambda)$ is the universal finite-dimensional $\tilde{\mathfrak{g}}$-module with highest $\ell$-weight $\lambda$; every simple object of $\widetilde{\mathscr{C}}$ is highest- $\ell$-weight.

Remark. It is not true that the module $V_{q}(\lambda)$ belongs to $\widetilde{\mathscr{C}}_{q}$ for every $\lambda \in \mathscr{P}_{q}^{+}$. This is so because $\mathbb{C}(q)$ is not algebraically closed. In fact, one can prove, using some results of Section 4.8 below, that $V_{q}(\lambda)$ is in $\widetilde{\mathscr{C}}_{q}$ if and only if $\lambda_{i}(u)$ splits in $\mathbb{C}(q)[u]$ for every $i \in I$. Otherwise, $V_{q}(\lambda)$ is quasi- $\ell$-weight in a sense analogous to that defined in [Jakelić and Moura 2009a] in the context of hyper loop algebras.

The following lemma is a consequence of the proof of Theorem 2.8.

Lemma 2.9. Let $V$ be a highest- $\ell$-weight module of $\tilde{\mathfrak{g}}$ and $v$ be a highest- $\ell$-weight vector. Then $V=U(\mathfrak{g}[t]) v$.

If $J \subseteq I$, we shall denote by $V_{q}\left(\lambda_{J}\right)$ the $U_{q}\left(\tilde{\mathfrak{g}}_{J}\right)$-irreducible module of highest $\ell$-weight $\lambda_{J}$. Similarly $V\left(\lambda_{J}\right)$ denotes the corresponding irreducible $\tilde{\mathfrak{g}}_{J}$-module. Similar notations for the Weyl modules are defined in the obvious way. 
Proposition 2.10 [Frenkel and Mukhin 2001]. For every $\lambda \in \mathscr{P}_{q}^{+}, V_{q}(\lambda)$ is a lowest$\ell$-weight module with lowest $\ell$-weight $\left(\lambda^{*}\right)^{-1}$. In particular, $V_{q}(\lambda)^{*} \cong V_{q}\left(\lambda^{*}\right)$.

2.11. Evaluation modules and Cartan involution. Given a g-module $V$, let $V(a)$ be the $\tilde{\mathfrak{g}}$-module obtained by pulling back the evaluation map ev ${ }_{a}$. Such modules are called evaluation modules. If $V=V(\lambda)$, we use the notation $V(\lambda, a)$ for the corresponding evaluation module.

Theorem 2.12 [Chari 1986; CP 1986; 2001]. Let $\lambda \in \mathscr{P P}^{+}$.

(a) If $\lambda=\omega_{\lambda, a}$ for some $\lambda \in P^{+}$and some $a \in \mathbb{C}^{\times}$, then $V(\lambda) \cong V(\lambda, a)$.

(b) If $\lambda=\prod_{j} \omega_{\lambda_{j}, a_{j}}$ as in (1-11), then

$$
V(\lambda) \cong \bigoplus_{j} V\left(\lambda_{j}, a_{j}\right) \quad \text { and } \quad W(\boldsymbol{\lambda}) \cong \bigoplus_{j} W\left(\boldsymbol{\omega}_{\lambda_{j}, a_{j}}\right)
$$

Corollary 2.13. Every object in $\widetilde{\mathscr{C}}$ is an $\ell$-weight module.

Assume $\mathfrak{g}$ is of type $A$ and consider the $\mathbb{C}(q)$-algebra $U_{q}^{\prime}(\mathfrak{g})$ given by generators $x_{i}^{ \pm}$and $k_{\mu}^{ \pm 1}$ with $i \in I, \mu \in P$, and the defining relations

$$
\begin{array}{cl}
k_{\mu} k_{\mu}^{-1}=k_{\mu}^{-1} k_{\mu}=1, \quad k_{\mu} k_{v}=k_{\mu+\nu}, \quad k_{\mu} x_{j}^{ \pm} k_{\mu}^{-1}=q^{\mu\left(h_{j}\right)} x_{j}^{ \pm}, \\
{\left[x_{i}^{+}, x_{j}^{-}\right]=\delta_{i, j} \frac{k_{\alpha_{i}}-k_{\alpha_{i}}^{-1}}{q-q^{-1}}, \quad \sum_{k=0}^{1-c_{i j}}(-1)^{k}\left(x_{i}^{ \pm}\right)^{\left(1-c_{i j}-k\right)} x_{j}^{ \pm}\left(x_{i}^{ \pm}\right)^{(k)}=0 \quad \text { if } i \neq j,}
\end{array}
$$

There is an obvious monomorphism of algebras $U_{q}(\mathfrak{g}) \rightarrow U_{q}^{\prime}(\mathfrak{g})$ taking $k_{i} \mapsto k_{\alpha_{i}}$. A $U_{q}^{\prime}(\mathfrak{g})$-module is said to be a weight module if the generators $k_{v}$ for $v \in P$ act diagonally with eigenvalues of the form $q^{(\nu, \mu)}$ for some $\mu \in P$, where $(\cdot, \cdot)$ is the bilinear form such that $\left(\alpha_{i}, \alpha_{j}\right)=c_{i j}$. It is not hard to see that restriction establishes an equivalence of categories from that of $U_{q}^{\prime}(\mathfrak{g})$-weight modules to $\mathscr{b}_{q}$. Henceforth we identify these two categories using this equivalence. The next proposition was proved in [Jimbo 1986, Section 2] and [CP 1994b, Proposition 3.4].

Proposition 2.14. Let $\mathfrak{g}$ be of type A. Then there exists an algebra homomorphism qev $: U_{q}(\tilde{\mathfrak{g}}) \rightarrow U_{q}^{\prime}(\mathfrak{g})$ such that if $\lambda \in P^{+}$and $V$ is the pullback of $V_{q}(\lambda)$ by qev, then there exists $l(\lambda) \in \mathbb{Z}$ such that $V$ is isomorphic to $V_{q}(\lambda)$, where

$$
\lambda=\prod_{i \in I} \omega_{i, a_{i}, \lambda\left(h_{i}\right)} \quad \text { with } a_{1}=q^{l(\lambda)} \text { and } \frac{a_{i+1}}{a_{i}}=q^{\lambda\left(h_{i}\right)+\lambda\left(h_{i+1}\right)+1} \text { for } i<n .
$$

Given $a \in \mathbb{C}(q)^{\times}$, there exists a unique $\mathbb{C}(q)$-algebra automorphism $\varrho_{a}$ of $U_{q}(\tilde{\mathfrak{g}})$ such that $\varrho_{a}$ is the identity on $U_{q}(\mathfrak{g})$ and $\varrho_{a}\left(x_{i, r}^{ \pm}\right)=a^{r} x_{i, r}^{ \pm}$. Let $\operatorname{qev}_{a}=$ qev $\circ \varrho_{a}$. 
Denote by $V_{q}(\lambda, a)$ the pullback of $V_{q}(\lambda)$ by the evaluation map qev ${ }_{a}$. It is easy to see that $V_{q}(\lambda, a) \cong V_{q}(\lambda)$ where

$$
\lambda=\prod_{i \in I} \omega_{i, a_{i}, \lambda\left(h_{i}\right)} \quad \text { with } a_{1}=a q^{l(\lambda)} \text { and } \frac{a_{i+1}}{a_{i}}=q^{\lambda\left(h_{i}\right)+\lambda\left(h_{i+1}\right)+1} \text { for } i<n .
$$

It turns out that, for $\mathfrak{g}$ not of type $A$, there is no analogue of the map qev. In fact, it is known (see [Chari 2001] for instance) that there exists $i \in I$ and $m \in \mathbb{Z}_{\geq 0}$ such that the action of $U_{q}(\mathfrak{g})$ on $V_{q}\left(m \omega_{i}\right)$ cannot be extended to one of $U_{q}(\tilde{\mathfrak{g}})$.

One easily checks that there exists a unique algebra involution $\tilde{\sigma}$ of $U_{q}(\tilde{\mathfrak{g}})$ such that $\tilde{\sigma}\left(x_{i, r}^{ \pm}\right)=x_{i,-r}^{\mp}, \tilde{\sigma}\left(k_{i}\right)=k_{i}^{-1}$, and $\tilde{\sigma}\left(h_{i, s}\right)=-h_{i,-s}$ for all $i \in I, r, s \in \mathbb{Z}$ and $s \neq 0$. The involution $\tilde{\sigma}$ is called the Cartan involution and it is also a coalgebra antiinvolution. The restriction of $\tilde{\sigma}$ to $U_{q}(\mathfrak{g})$ defines an involution $\sigma$ of $U_{q}(\mathfrak{g})$ also called the Cartan involution. Given a $U_{q}(\tilde{\mathfrak{g}})$-module $V$, let $V^{\tilde{\sigma}}$ be the pullback of $V$ by $\tilde{\sigma}$. Similarly, $V^{\sigma}$ will denote the pullback of a $U_{q}(\mathfrak{g})$-module $V$ by $\sigma$. It is not difficult to see that a highest- $\ell$-weight vector of $V_{q}(\lambda)$ is a lowest- $\ell$-weight vector of $V_{q}(\lambda)^{\tilde{\sigma}}$. Moreover, it follows from (1-3) that

$$
\tilde{\sigma}\left(\Lambda_{i}^{ \pm}(u)\right)=\left(\Lambda_{i}^{\mp}(u)\right)^{-1}, \quad \text { where } \Lambda_{i}^{ \pm}(u)=\sum_{r=0}^{\infty} \Lambda_{i, \pm r} u^{r}
$$

and the inverse is that of formal power series in $u$. It is now not difficult to complete the proof of the next proposition.

Proposition 2.15. Let $\lambda \in P^{+}$and $\lambda \in \mathscr{P}_{q}^{+}$. Then

$$
V_{q}(\lambda)^{\sigma} \cong V_{q}\left(\lambda^{*}\right) \text { and } V_{q}(\lambda)^{\tilde{\sigma}} \cong V_{q}\left({ }^{*} \lambda\right) .
$$

The analogous result in the classical case is established similarly.

We end this subsection with a remark. Let $\mathfrak{g}$ be of type $A$, let $\lambda \in \mathscr{P}_{q}^{+}$be such that $V_{q}(\lambda) \cong V_{q}(\lambda, a)$ for some $a \in \mathbb{C}(q)^{\times}$, and set $b_{n}=\left(a q^{l(\lambda)+n+1}\right)^{-1}$. Then

$$
{ }^{*} \lambda=\prod_{i \in I} \omega_{i, b_{i}, \lambda^{*}\left(h_{i}\right)} \quad \text { with } \frac{b_{i}}{b_{i-1}}=q^{-\left(\lambda^{*}\left(h_{i}\right)+\lambda^{*}\left(h_{i-1}\right)+1\right)} \text { for } i>1 .
$$

\subsection{Classical limits.}

Definition 2.17. Denote by $\mathscr{P}_{\mathrm{A}}^{+}$the subset of $\mathscr{P}_{q}$ consisting of $n$-tuples of polynomials with coefficients in $\mathbb{A}$. Let also $\mathscr{P}_{\mathbb{A}}^{++}$be the subset of $\mathscr{P}_{\mathbb{A}}^{+}$consisting of $n$-tuples of polynomials whose leading terms are in $\mathbb{C} q^{\mathbb{Z}} \backslash\{0\}=\mathbb{A}^{\times}$. Given $\lambda \in \mathscr{P}_{\mathbb{A}}^{+}$, let $\bar{\lambda}$ be the element of $\mathscr{P}^{+}$obtained from $\lambda$ by evaluating $q$ at 1 .

Recall that an $\mathbb{A}$-lattice (or form) of a $\mathbb{C}(q)$-vector space $V$ is a free $\mathbb{A}$-submodule $L$ of $V$ such that $\mathbb{C}(q) \otimes_{\mathbb{A}} L=V$. If $V$ is a $U_{q}(\tilde{\mathfrak{g}})$-module, a $U_{\mathbb{A}}(\tilde{\mathfrak{g}})$-admissible 
lattice of $V$ is an $\mathbb{A}$-lattice of $V$ that is also a $U_{\mathbb{A}}(\tilde{\mathfrak{g}})$-submodule of $V$. Given a $U_{\mathbb{A}}(\tilde{\mathfrak{g}})$-admissible lattice of a $U_{q}(\tilde{\mathfrak{g}})$-module $V$, define

$$
\bar{L}=\mathbb{C} \otimes_{\mathbb{A}} L,
$$

where $\mathbb{C}$ is regarded as an $\mathbb{A}$-module by letting $q$ act as 1 . Then $\bar{L}$ is a $\tilde{\mathfrak{g}}$-module by Proposition 1.4 and $\operatorname{dim}(\bar{L})=\operatorname{dim}(V)$. The next theorem is essentially a corollary of the proof of Theorem 2.8.

Theorem 2.18. Let $V$ be a nontrivial quotient of $W_{q}(\lambda)$ for some $\lambda \in \mathscr{P}_{\mathbb{A}}^{++}$, let $v$ be a highest- $\ell$-weight vector of $V$, and let $L=U_{\mathbb{A}}(\tilde{\mathfrak{g}}) v$. Then $L$ is a $U_{\mathbb{A}}(\tilde{\mathfrak{g}})$-admissible lattice of $V$ and $\operatorname{ch}(\bar{L})=\operatorname{ch}(V)$. In particular, $\bar{L}$ is a quotient of $W(\bar{\lambda})$.

Definition 2.19. Let $\lambda \in \mathscr{P}_{\mathrm{A}}^{++}$, let $v$ be a highest- $\ell$-weight vector of $V_{q}(\lambda)$, and let $L=U_{\text {A }}(\tilde{\mathfrak{g}}) v$. We denote by $\overline{V_{q}(\lambda)}$ the $\tilde{\mathfrak{g}}$-module $\bar{L}$.

We shall also use the following straightforward lemma.

Lemma 2.20. Suppose $V$ and $V^{\prime}$ are $U_{q}(\tilde{\mathfrak{g}})$-modules, and $L$ and $L^{\prime}$ are $U_{\mathbb{A}}(\tilde{\mathfrak{g}})$ submodules. Suppose $\phi: V \rightarrow V^{\prime}$ is a $U_{q}(\tilde{\mathfrak{g}})$-module map such that $\phi(L) \subseteq L^{\prime}$. Then $\bar{\phi}:=1 \otimes \phi: \bar{L} \rightarrow \bar{L}^{\prime}$ is a $\tilde{\mathfrak{g}}$-module map.

\section{Minimal affinizations}

3.1. Classification. Chari [1995] introduced the notion of minimal affinizations of an irreducible $U_{q}(\mathfrak{g})$-module, as follows.

Given $\lambda \in P^{+}$, an object $V \in \widetilde{\mathscr{C}}_{q}$ is said to be an affinization of $V_{q}(\lambda)$ if, as a $U_{q}(\mathfrak{g})$-module, $V \cong V_{q}(\lambda) \oplus \bigoplus_{\mu<\lambda} V_{q}(\mu)^{\oplus m_{\mu}(V)}$ for some $m_{\mu}(V) \in \mathbb{Z}_{\geq 0}$. We say two affinizations of $V_{q}(\lambda)$ are equivalent if they are isomorphic as $U_{q}(\mathfrak{g})$-modules. If $\lambda \in \mathscr{P}_{q}^{+}$is such that $\operatorname{wt}(\lambda)=\lambda$, then $V_{q}(\lambda)$ is clearly an affinization of $V_{q}(\lambda)$. The partial order on $P^{+}$induces a natural partial order on the set of (equivalence classes of) affinizations of $V_{q}(\lambda)$. Namely, if $V$ and $W$ are affinizations of $V_{q}(\lambda)$, we say that $V \leq W$ if either $m_{\mu}(V) \leq m_{\mu}(W)$ for all $\mu \in P^{+}$or if for all $\mu \in P^{+}$ such that $m_{\mu}(V)>m_{\mu}(W)$, there exists $v>\mu$ such that $m_{v}(V)<m_{v}(W)$. A minimal element of this partial order is said to be a minimal affinization.

Suppose $\mathfrak{g}$ is not of types $D$ or $E$. Given $\lambda \in \mathscr{P}_{q}^{+}$, set

$$
\lambda^{\circ}=\lambda^{*} \quad \text { if } \mathfrak{g}=\mathfrak{s l}_{n+1} \text { and } \lambda^{\circ}={ }^{*} \lambda \text { otherwise. }
$$

Recall that, in these cases, $\lambda^{*}=\lambda$ for all $\lambda \in P^{+}$except if $\mathfrak{g}$ is of type $A$.

The following main result of [Chari 1995; CP 1996b; 1995] gives a partial classification of the highest $\ell$-weights of the minimal affinizations, and gives the complete classification when $\mathfrak{g}$ is not of types $D$ or $E$.

Theorem 3.2. Let $\lambda \in \mathscr{P}_{q}^{+}, \lambda=\operatorname{wt}(\lambda)$, and $V=V_{q}(\lambda)$. Suppose $\mathfrak{g}$ is not of types $D$ or $E$. Then $V$ is a minimal affinization of $V_{q}(\lambda)$ if and only if $V^{*}$ and $V^{\tilde{\sigma}}$ are 
minimal affinizations of $V_{q}\left(\lambda^{*}\right)$. In that case, there exist $a \in \mathbb{C}(q)^{\times}$and $\mu \in\left\{\lambda, \lambda^{*}\right\}$ such that either $\lambda$ or $\lambda^{\circ}$ is equal to

$$
\prod_{i=1}^{n} \omega_{i, a_{i}, \mu\left(h_{i}\right)} \quad \text { with } a_{1}=a \text { and } \frac{a_{i+1}}{a_{i}}=q^{d_{i} \mu\left(h_{i}\right)+d_{i+1} \mu\left(h_{i+1}\right)+r_{i}^{\vee}}
$$

for all $i \in I, i<n$, where $r_{i}^{\vee}=d_{i}-1-c_{i, i+1}$. Equivalently, $V$ is a minimal affinization of $V_{q}(\lambda)$ if and only if there exist $a \in \mathbb{C}(q)^{\times}$and $\epsilon \in\{1,-1\}$ such that

$$
\lambda=\prod_{i=1}^{n} \omega_{i, a_{i}, \lambda\left(h_{i}\right)} \quad \text { with } a_{1}=a \text { and } \frac{a_{i+1}}{a_{i}}=q^{\epsilon\left(d_{i} \lambda\left(h_{i}\right)+d_{i+1} \lambda\left(h_{i+1}\right)+r_{i}^{\vee}\right)}
$$

for all $i \in I$ with $i<n$. If $\mathfrak{g}$ is of type $D$ or $E$, suppose the support of $\lambda$ is contained in a connected subdiagram $J \subseteq I$ of type $A$. Then $V$ is a minimal affinization of $V_{q}(\lambda)$ if and only if $V_{q}\left(\lambda_{J}\right)$ is a minimal affinization of $V_{q}\left(\lambda_{J}\right)$.

The next corollary is immediate (recall from Section 1.1 that $\overline{\operatorname{supp}}(\lambda)$ is the minimal connected subdiagram of $I$ containing $\operatorname{supp}(\lambda)$ ).

Corollary 3.3. If $\lambda \in P^{+}$is such that $\overline{\operatorname{supp}}(\lambda)$ does not contain a subdiagram of type $D_{4}$, then $V_{q}(\lambda)$ has a unique equivalence class of minimal affinizations.

Remark. We warn that the conditions we give in Theorem 3.2 do not match the ones given in [Chari 1995; CP 1996b; 1995], due to different normalizations in some definitions. Our notation follows more closely that of [Hernandez 2007], which is more uniform. We also note that $r_{i}^{\vee}=d_{i+1}-c_{i+1, i}$ and $r_{i}^{\vee} \in\left\{r^{\vee}-1, r^{\vee}\right\}$ for all $i \in I$ with $i<n$. It is easy to check that $r_{i}^{\vee}=r^{\vee}$ for all $i<n$ if $\mathfrak{g}$ is of types $A, B$, or $G$. If $\mathfrak{g}$ is of type $C$, then $r_{i}^{\vee}=r^{\vee}-1$ if and only if $i<n-1$. Finally, if $\mathfrak{g}$ is of type $F$, then $r_{i}^{\vee}=r^{\vee}$ if and only if $\alpha_{i}$ is a long root.

Corollary 3.4. For every $a \in \mathbb{C}(q)^{\times}, i \in I$ and $m \in \mathbb{Z}_{\geq 0}$, the module $V_{q}\left(\omega_{i, a, m}\right)$ is a minimal affinization of $V_{q}\left(m \omega_{i}\right)$.

The modules $V_{q}\left(\omega_{i, a, m}\right)$ are known as Kirillov-Reshetikhin modules.

In the cases not covered by Theorem 3.2, that is, when $\overline{\operatorname{supp}}(\lambda)$ contains a subdiagram of type $D_{4}$, then $V_{q}(\lambda)$ may have more then one equivalence class of minimal affinizations; see [CP 1996b; 1996a]. We shall briefly discuss these cases in Sections 5.11 and 5.18.

We now state a few results that were used in the proof of Theorem 3.2 and will be useful for us as well. The proofs can be found in [CP 1996b].

Lemma 3.5. Suppose $\varnothing \neq J \subseteq I$ is a connected subdiagram of the Dynkin diagram of $\mathfrak{g}$. Let $V=V_{q}(\lambda)$, let $v$ be a highest- $\ell$-weight vector of $V$, and let $V_{J}=U_{q}\left(\tilde{\mathfrak{g}}_{J}\right) v$. Then $V_{J} \cong V_{q}\left(\lambda_{J}\right)$. 
Definition 3.6. Suppose $\mathfrak{g}$ is not of type $D$ or $E$. A connected subdiagram $J \subseteq I$ is said to be admissible if $J$ is of type $A$. If $\mathfrak{g}$ is of type $D$ or $E$, let $i_{0} \in I$ be the unique element connected to three other nodes. A connected subdiagram $J \subseteq I$ is said to be admissible if $J$ is of type $A$ and $J \backslash\left\{i_{0}\right\}$ is connected.

Proposition 3.7. Suppose $J \subseteq I$ is admissible and that $\lambda \in \mathscr{P}_{q}^{+}$is such that $V_{q}(\lambda)$ is a minimal affinization of $V_{q}(\lambda)$, where $\lambda=\mathrm{wt}(\lambda)$. Then $V_{q}\left(\lambda_{J}\right)$ is a minimal affinization of $V_{q}\left(\lambda_{J}\right)$.

Proposition 3.8. Let $\lambda \in P_{q}^{+}$and $\lambda=\operatorname{wt}(\lambda)$. If $V_{q}(\lambda)$ is a minimal affinization of $V_{q}(\lambda)$, then there exist $a_{i} \in \mathbb{C}(q)^{\times}$for $i \in I$ such that $\lambda=\prod_{i \in I} \omega_{i, a_{i}, \lambda\left(h_{i}\right)}$ and $a_{i} / a_{j} \in q^{\mathbb{Z}}$ for all $i, j \in I$.

Proof. The existence of $a_{i} \in \mathbb{C}(q)^{\times}$for $i \in I$ such that $\lambda=\prod_{i \in I} \omega_{i, a_{i}, \lambda\left(h_{i}\right)}$ follows from Proposition 3.7 and the classification of minimal affinizations for $\mathfrak{s l}_{2}$. The condition $a_{i} / a_{j} \in q^{\mathbb{Z}}$ for all $i, j \in I$ can be proved from results of [Chari 2002] (see Section 4.1 below). Alternatively, the proposition is immediate from Theorems 3.2 and 5.12 in the cases covered by them.

Corollary 3.9. For every $\lambda \in P^{+}$, there exist $\lambda \in \mathscr{P}_{\mathbb{A}}^{++}$such that $V_{q}(\lambda)$ is a minimal affinization of $V_{q}(\lambda)$.

\subsection{Restricted limits.}

Definition 3.11. Let $V$ be a $\mathbb{Z}_{\geq 0}$-graded vector space and denote its $s$-th graded piece by $V[s]$. A $\mathfrak{g}[t]$-module $V$ is said to be $\mathbb{Z}_{\geq 0}$-graded if $V$ is a $\mathbb{Z}_{\geq 0}$-graded vector space and $x \otimes t^{r} v \in V[r+s]$ for every $v \in V[s], x \in \mathfrak{g}$, and $r, s \in \mathbb{Z}_{\geq 0}$. A $\mathbb{Z}_{\geq 0}$-graded $\mathfrak{g}[t]$-module $V$ satisfying $V[r]=0$ for $r \gg 0$ is said to be a restricted $\mathfrak{g}[t]$-module. If $V$ is a $\mathbb{Z}_{\geq 0}$-graded $\mathfrak{g}[t]$-module, denote by $V(s)$ the quotient of $V$ by its $\mathfrak{g}[t]$-submodule $\bigoplus_{k>s} V[k]$.

The next lemma follows immediately from Proposition 3.8.

Lemma 3.12. Suppose $\lambda \in \mathscr{P}_{\mathbb{A}}^{++}$is such that $V_{q}(\lambda)$ is a minimal affinization. Then $\bar{\lambda}=\omega_{\lambda, a}$ for some $a \in \mathbb{C}^{\times}$, where $\lambda=\operatorname{wt}(\lambda)$.

Proposition 3.13. Suppose $\lambda \in \mathscr{P}_{\mathbb{A}}^{++}$is such that $V_{q}(\lambda)$ is a minimal affinization and that $J \subseteq I$ is an admissible subdiagram. Let $v$ be a highest- $\ell$-weight vector of $V=\overline{V_{q}(\lambda)}$, let $\lambda=\operatorname{wt}(\lambda)$, and let $a \in \mathbb{C}^{\times}$be such that $\bar{\lambda}=\omega_{\lambda, a}$. Then $x_{\alpha, r}^{-} v=a^{r} x_{\alpha}^{-} v$ for every $\alpha \in R_{J}^{+}$.

Proof. Let $J$ be admissible, let $\alpha \in R_{J}^{+}$, and let $V_{J}=U_{q}\left(\tilde{\mathfrak{g}}_{J}\right) v^{\prime}$, where $v^{\prime} \in V_{q}(\lambda)$ is such that $\overline{v^{\prime}}=v$. Then $V_{J}$ is a minimal affinization by Proposition 3.7 and, since $J$ is of type $A, V_{J}$ is irreducible as a $U_{q}\left(\mathfrak{g}_{J}\right)$-module by Theorem 3.2. Hence, the $\tilde{\mathfrak{g}}_{J}$-submodule of $V$ generated by $v$ is isomorphic to $V\left(\lambda_{J}, a\right)$.

Recall the definition of the maps $\tau_{a}: \mathfrak{g}[t] \rightarrow \mathfrak{g}[t]$ from Section 1.1. 
Definition 3.14. Let $\lambda \in \mathscr{P}_{\mathrm{A}}^{++}$, let $\lambda=\operatorname{wt}(\lambda)$, and let $a \in \mathbb{C}^{\times}$be such that $\bar{\lambda}=\omega_{\lambda, a}$. The $\mathfrak{g}[t]$-module $L(\lambda)$ is defined to be the pullback of $\overline{V_{q}(\lambda)}$ by $\tau_{a}$. Define also the module $A(\lambda)$ to be the $\mathfrak{g}[t]$-module given by the quotient of $U(\mathfrak{g}[t])$ by the left ideal generated by

$$
\mathfrak{n}^{+}[t], \quad \mathfrak{h} \otimes t \mathbb{C}[t], \quad h_{i}-\lambda\left(h_{i}\right), \quad\left(x_{\alpha_{i}}^{-}\right)^{\lambda\left(h_{i}\right)+1}, \quad x_{\alpha, 1}^{-}
$$

for all $i \in I$ and all $\alpha \in R_{J}^{+}$for some admissible subdiagram $J \subseteq I$. Denote by $v_{\lambda}$ the image of 1 in $A(\lambda)$, so that $A(\lambda)=U\left(\mathfrak{n}^{-}[t]\right) v_{\lambda}$.

It immediately follows from Theorem 2.12, Proposition 3.13, and Lemma 2.9 that $L(\lambda)$ is a quotient of $A(\lambda)$. It is also clear that $A(\lambda)$ is a $\mathbb{Z}_{\geq 0}$-graded $\mathfrak{g}[t]-$ module. We call the module $L(\lambda)$ the restricted limit of $V_{q}(\lambda)$. It is immediate from Theorem 2.18 that

$$
\operatorname{ch}(L(\lambda))=\operatorname{ch}\left(V_{q}(\lambda)\right) .
$$

When $\lambda=m \omega_{i}$ for some $m \in \mathbb{Z}_{\geq 0}$ and some $i \in I$, the modules $L\left(\omega_{i, a, m}\right)$ are the restricted Kirillov-Reshetikhin modules of highest-weight $m \omega_{i}$. Chari and Moura studied these for $\mathfrak{g}$ of classical type in [2006] and for $\mathfrak{g}$ of type $G_{2}$ in [2007].

Proposition 3.15. For every $\lambda \in P^{+}$, the module $A(\lambda)$ is finite-dimensional. In particular, $A(\lambda)$ is restricted.

Proof. Since $A(\lambda)=U\left(\mathfrak{n}^{-}[t]\right) v_{\lambda}$, it immediately follows that $(A(\lambda)[r])_{\mu}$ is finitedimensional for every $r \in \mathbb{Z}_{\geq 0}$ and every $\mu \in P$. The relations $\left(x_{\alpha_{i}}^{-}\right)^{\lambda\left(h_{i}\right)+1} v_{\lambda}=0$ for all $i \in I$ implies, as usual, that the elements $x_{\alpha_{i}}^{ \pm}$act locally nilpotently on $A(\lambda)$ and hence $\operatorname{dim}\left(A(\lambda)_{\mu}\right)=\operatorname{dim}\left(A(\lambda)_{w \mu}\right)$ for every $\mu \in P$ and $w \in \mathcal{W}$. This in turn implies that $A(\lambda)_{\mu} \neq 0$ if and only if $w_{0} \lambda \leq \mu \leq \lambda$. Hence $A(\lambda)$ has only finitelymany nontrivial weight spaces. Using the defining relations of $A(\lambda)$ together with basic commutation relations in $\mathfrak{g}[t]$, it is trivial to see that $x_{\alpha, r}^{-} v_{\lambda}=0$ for all $\alpha \in R^{+}$ if $r \gg 0$. This together with the PBW theorem then implies that $(A(\lambda)[s])_{\mu}=0$ for every $\mu \in P$ if $s \gg 0$. In fact, let $r \in \mathbb{Z}_{\geq 0}$ be such that $x_{\alpha, s}^{-} v_{\lambda}=0$ for all $\alpha \in R^{+}$ and all $s \geq r$. Fix a total order on $R^{+} \times \mathbb{Z}_{\geq 0}$ such that $(\alpha, k)<(\beta, l)$ whenever $k<r$ and $l \geq r$. The PBW monomials for $U\left(\mathfrak{n}^{-}[t]\right)$ are then formed such that $x_{\beta, l}^{-}$ occur to the right of $x_{\alpha, k}^{-}$whenever $(\alpha, k)<(\beta, l)$. Hence, to get to the $s$-th graded piece of $A(\lambda)$ with $s \gg r$, one would have to apply elements of the form $x_{\alpha, k}^{-}$with $k<r$ to $v_{\lambda}$ "too many times", and the maximal possible weight of $A(\lambda)[s]$ would fall out of the set of weights lying in between $w_{0} \lambda$ and $\lambda$.

3.16. Relations for $\boldsymbol{L}(\lambda)$. We now state our main results and conjectures.

Definition 3.17. Let $m \in \mathbb{Z}_{\geq 0}$ and $i \in I$. The $\mathfrak{g}[t]$-module $M\left(m \omega_{i}\right)$ is the quotient of $U(\mathfrak{g}[t])$ by the left ideal generated by

$$
\mathfrak{n}^{+}[t], \quad \mathfrak{h} \otimes t \mathbb{C}[t], \quad h_{j}, \quad h_{i}-m, \quad x_{\alpha_{j}}^{-}, \quad\left(x_{\alpha_{i}}^{-}\right)^{m+1}, \quad x_{\alpha_{i}, 1}^{-} \quad \text { for all } j \neq i .
$$


Quite clearly $M\left(m \omega_{i}\right)$ is a $\mathbb{Z}_{\geq 0}$-graded $\mathfrak{g}[t]$-module, and $A\left(m \omega_{i}\right)$ is a quotient of $M\left(m \omega_{i}\right)$. The next proposition follows from [Chari 2001; CM 2006; 2007].

Proposition 3.18. Suppose $\mathfrak{g}$ is not of type $E$ or $F$. Let $i \in I, m \in \mathbb{Z}_{\geq 0}$, and $a \in \mathbb{C}^{\times}$.

(a) There exists $b_{i} \in\{1,2,3\}$ such that, if $m=m_{1} b_{i}+m_{0}$ with $0 \leq m_{0}<b_{i}$ and $T\left(m \omega_{i}\right)$ is the $\mathfrak{g}[t]$-submodule of $M\left(b_{i} \omega_{i}\right)^{\otimes m_{1}} \otimes M\left(m_{0} \omega_{i}\right)$ generated by the top weight space, then $M\left(m \omega_{i}\right) \cong T\left(m \omega_{i}\right)$.

(b) $M\left(m \omega_{i}\right) \cong A\left(m \omega_{i}\right) \cong L\left(\omega_{i, a, m}\right)$.

Our goal is to generalize the proposition above for minimal affinizations other than Kirillov-Reshetikhin modules. Let us introduce notation, as follows. Given $i \in I$ and $m, r \in \mathbb{Z}_{\geq 0}$, let $v_{i, m}$ be the image of 1 in $M\left(m \omega_{i}\right)$ and set

$$
R^{+}(i, m, r)=\left\{\alpha \in R^{+}: x_{\alpha, r}^{-} v_{i, m}=0\right\} .
$$

Since $(\mathfrak{h} \otimes t \mathbb{C}[t]) v_{i, m}=0$, it follows that

$$
R^{+}(i, m, r) \subseteq R^{+}(i, m, s) \text { for all } s \geq r .
$$

The sets $R^{+}(i, m, r)$ for $\mathfrak{g}$ not of types $E$ and $F$ were explicitly described in [Chari 2001; CM 2006; 2007]. We will eventually write them down precisely. For the moment, let us just observe that $R^{+}(i, m, r)=R^{+}$if $r \gg 0$ since $A\left(m \omega_{i}\right)$ is restricted. In fact, if $\mathfrak{g}$ is of classical type, then $R(i, m, 2)=R^{+}$for every $i \in I$ and $m \in \mathbb{Z}_{\geq 0}$. Observe also that $R^{+}(i, 0,0)=R^{+}$for all $i \in I$ since $L(0)$ is the trivial representation. Now, given $\lambda \in P^{+}$and $r \in \mathbb{Z}_{\geq 0}$, set

$$
R^{+}(\lambda, r)=\bigcap_{i \in I} R^{+}\left(i, \lambda\left(h_{i}\right), r\right)
$$

Since $R^{+}(j, 0, s)=R^{+}$for all $j \in I$ and $s \in \mathbb{Z}_{\geq 0}$, it follows that $R^{+}\left(m \omega_{i}, r\right)=$ $R^{+}(i, m, r)$ for all $i \in I$ and $m, r \in \mathbb{Z}_{\geq 0}$ and that

$$
R^{+}(\lambda, r)=R^{+} \quad \text { if } r \gg 0 .
$$

Definition 3.19. Given $\lambda \in P^{+}$, let $M(\lambda)$ be the $\mathfrak{g}[t]$-module given by the quotient of $U(\mathfrak{g}[t])$ by the left ideal generated by

$$
\mathfrak{n}^{+}[t], \quad \mathfrak{h} \otimes t \mathbb{C}[t], \quad h_{i}-\lambda\left(h_{i}\right), \quad\left(x_{\alpha_{i}}^{-}\right)^{\lambda\left(h_{i}\right)+1}, \quad x_{\alpha, r}^{-}
$$

for all $i \in I, \quad r \in \mathbb{Z}_{\geq 0}$, and $\alpha \in R^{+}(\lambda, r)$. Let $T(\lambda)$ be the $\mathfrak{g}[t]$-submodule of $\bigoplus_{i \in I} M\left(\lambda\left(h_{i}\right) \omega_{i}\right)$ generated by the top weight space.

Definitions 3.17 and 3.19 of $M\left(m \omega_{i}\right)$ coincide since $R^{+}\left(m \omega_{i}, r\right)=R^{+}(i, m, r)$ for all $i \in I$ and $m, r \in \mathbb{Z}_{\geq 0}$. The modules $M(\lambda)$ are clearly $\mathbb{Z}_{\geq 0}$-graded. It follows from Proposition 3.13 that $M(\lambda)$ is a quotient of $A(\lambda)$ and hence a restricted $\mathfrak{g}[t]-$ module. Also, $T(\lambda)$ is clearly a restricted quotient of $M(\lambda)$ by Proposition 3.18. 
The following is what we expect to be the generalization of Proposition 3.18 when $\mathfrak{g}$ and $\lambda$ are as in Theorem 3.2.

Conjecture 3.20. Let $\lambda \in P^{+}$be such that $\overline{\operatorname{supp}}(\lambda)$ does not contain a subdiagram of type $D_{4}$, and suppose $\lambda \in \mathscr{P}_{\mathrm{A}}^{++}$is such that $V_{q}(\lambda)$ is a minimal affinization of $V_{q}(\lambda)$. Then, $T(\lambda) \cong M(\lambda) \cong L(\lambda)$.

Proposition 3.18 says the conjecture holds when $\lambda$ is a multiple of a fundamental weight and $\mathfrak{g}$ is not of type $E$ or $F$. It is quite simple to see that the conjecture also holds when $\mathfrak{g}$ is of type $A$ for general $\lambda \in P^{+}$. We now state our main partial results in the direction of proving Conjecture 3.20.

Proposition 3.21. Let $\lambda \in \mathscr{P}_{\mathbb{A}}^{++}$be such that $V_{q}(\lambda)$ is a minimal affinization of $V_{q}(\lambda)$, where $\lambda=\operatorname{wt}(\lambda)$. Then, $T(\lambda)$ is a quotient of $L(\lambda)$.

Proposition 3.22. Let $\lambda \in P^{+}$be such that $\overline{\operatorname{supp}}(\lambda)$ does not contain a subdiagram of type $D_{4}$, and suppose $\mathfrak{g}$ is orthogonal. Then, $L(\lambda)$ is a quotient of $M(\lambda)$.

Corollary 3.23. In the conditions of Proposition 3.22, the first isomorphism of Conjecture 3.20 implies the second.

Proposition 3.24. Conjecture 3.20 holds in the following cases:

(a) $\mathfrak{g}$ is of type $B$ and $\operatorname{supp}(\lambda) \subseteq\{1,2,3, n\}$ with $\lambda\left(h_{n}\right) \leq 1$ if $n>3$.

(b) $\mathfrak{g}$ is of type $D$ and $\operatorname{supp}(\lambda) \subseteq(\{1,2,3\} \cap J) \cup\{m\}$ with $m \in\{n-1, n\}$. Here $J=I \backslash\{n-1, n\}$.

(c) $\mathfrak{g}$ is of type $D$ and $\operatorname{supp}(\lambda) \subseteq\{n-2, n-1, n\}$.

In proving Proposition 3.24, we obtain character formulas for $M(\lambda)$. The proofs of Propositions 3.21 and 3.22 are given in Sections 4.1 and 4.15, respectively. Proposition 3.24 is proved in Sections 5.3 and 5.8.

Remark. If $\mathfrak{g}$ is of classical type, then $R^{+}(\lambda, 2)=R^{+}$for every $\lambda \in P^{+}$since $R(i, m, 2)=R^{+}$for every $i \in I$ and $m \in \mathbb{Z}_{\geq 0}$, as mentioned previously. This implies that the modules $M(\lambda)$ can be regarded as modules for the truncated algebra $\mathfrak{g}[t] /\left(\mathfrak{g} \otimes t^{2} \mathbb{C}[t]\right)$ in this case. This was what motivated Chari and Greenstein [2009] to study the relations between the finite-dimensional representation theory of $U_{q}(\tilde{\mathfrak{g}})$ and Koszul algebras. We shall leave the discussion of how our methods are related to theirs to a forthcoming publication.

\section{Tensor products}

4.1. Tensor products of Kirillov-Reshetikhin modules. The goal of this section is to prove Proposition 3.21. The following fact is easily established from (1-7).

Proposition 4.2. Let $\lambda, \boldsymbol{\mu} \in \mathscr{P}_{q}^{+}$. Then, the $U_{q}(\tilde{\mathfrak{g}})$-submodule of $V_{q}(\lambda) \otimes V_{q}(\boldsymbol{\mu})$ generated by the top weight space is a quotient of $W_{q}(\lambda \mu)$. 
The following proposition follows from the results of [Chari 2002].

Proposition 4.3. Let $l \in \mathbb{Z}_{\geq 1}, i_{j} \in I, m_{j} \in \mathbb{Z}_{\geq 1}$, and $a_{j} \in \mathbb{C}(q)^{\times}$for $j=1, \ldots$, l. If $a_{j} / a_{k} \notin q^{\mathbb{Z}>0}$ for $j>k$, then $V_{q}\left(\omega_{i_{1}, a_{1}, m_{1}}\right) \otimes \cdots \otimes V_{q}\left(\omega_{i_{l}, a_{l}, m_{l}}\right)$ is a highest- $\ell$-weight module.

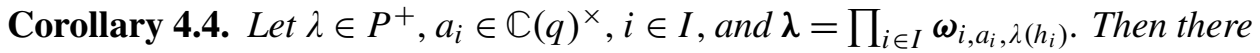
is an ordering $i_{1}, \ldots, i_{n}$ of I such that $V_{q}(\lambda)$ is isomorphic to the $U_{q}(\tilde{\mathfrak{g}})$-submodule of $V_{q}\left(\omega_{i_{1}, a_{i_{1}}, \lambda\left(h_{i_{1}}\right)}\right) \otimes \cdots \otimes V_{q}\left(\omega_{i_{n}, a_{i_{n}}, \lambda\left(h_{i_{n}}\right)}\right)$ generated by the top weight space.

Proof. Let $\omega \in \mathscr{P}_{q}^{+}$be such that $\omega^{*}=\lambda$, and write $\omega_{i}(u)=\omega_{i, b_{i}, \lambda^{*}\left(h_{i}\right)}$ for some $b_{i} \in \mathscr{P}_{q}^{+}$. Let also $i^{\prime}=w_{0} \cdot i$ for all $i \in I$. It follows from Proposition 4.3 that there is an ordering $i_{1}, \ldots, i_{n}$ of $I$ such that

$$
V:=V_{q}\left(\omega_{i_{n}^{\prime}}, b_{i_{n}^{\prime}}, \lambda^{*}\left(h_{i_{n}^{\prime}}\right)\right) \otimes \cdots \otimes V_{q}\left(\omega_{i_{1}^{\prime}, b_{i_{1}^{\prime}}, \lambda^{*}\left(h_{i_{1}^{\prime}}\right)}\right)
$$

is highest- $\ell$-weight. Let $W$ be the proper maximal submodule of $V$. Thus, we have a short exact sequence $0 \rightarrow W \rightarrow V \rightarrow V_{q}(\omega) \rightarrow 0$. Then, by Propositions 2.10 and 1.7, we also have the short exact sequence

$$
0 \rightarrow V_{q}(\lambda) \rightarrow V_{q}\left(\omega_{i_{1}, a_{i_{1}}, \lambda\left(h_{i_{1}}\right)}\right) \otimes \cdots \otimes V_{q}\left(\omega_{i_{n}, a_{i_{n}}, \lambda\left(h_{i_{n}}\right)}\right) \rightarrow W^{*} \rightarrow 0,
$$

since $V^{*} \cong V_{q}\left(\omega_{i_{1}, a_{i_{1}}, \lambda\left(h_{i_{1}}\right)}\right) \otimes \cdots \otimes V_{q}\left(\omega_{i_{n}, a_{i_{n}}, \lambda\left(h_{i_{n}}\right)}\right)$ and $V_{q}(\omega)^{*} \cong V_{q}(\lambda)$.

Proof of Proposition 3.21. Given $i \in I$, let $a_{i} \in \mathbb{A}^{\times}$be such that $\lambda=\prod_{i \in I} \omega_{i, a_{i}, \lambda\left(h_{i}\right)}$ and let $v_{i}$ be a highest- $\ell$-weight vector of $V\left(\omega_{i, a_{i}, \lambda\left(h_{i}\right)}\right)$. Let also $i_{1}, \ldots, i_{n}$ be an ordering of $I$ as in Corollary 4.4, and let

$$
v=v_{i_{1}} \otimes \cdots \otimes v_{i_{n}} \in V_{q}\left(\boldsymbol{\omega}_{i_{1}, a_{i_{1}}, \lambda\left(h_{i_{1}}\right)}\right) \otimes \cdots \otimes V_{q}\left(\boldsymbol{\omega}_{i_{n}, a_{i_{n}}, \lambda\left(h_{i_{n}}\right)}\right) .
$$

Consider $L_{i}=U_{\mathbb{A}}(\tilde{\mathfrak{g}}) v_{i}, L=U_{\mathbb{A}}(\tilde{\mathfrak{g}}) v$, and $L^{\prime}=L_{i_{1}} \otimes \cdots \otimes L_{i_{n}}$. Let $a \in \mathbb{C}^{\times}$be such that $\bar{\lambda}=\boldsymbol{\omega}_{\lambda, a}$, and observe that $L(\lambda) \cong \tau_{a}^{*}(\bar{L})$ and $M\left(\lambda\left(h_{i}\right) \omega_{i}\right) \cong \tau_{a}^{*}\left(\overline{L_{i}}\right)$, where $\tau_{a}^{*} K$ denotes the pullback of a $\mathfrak{g}[t]$-module $K$ by $\tau_{a}$. Moreover, it is easy to see that $L \subseteq L^{\prime}$ and that $\overline{L^{\prime}} \cong \overline{L_{i_{1}}} \otimes \cdots \otimes \overline{L_{i_{n}}}$.

Let $\bar{\phi}: \bar{L} \rightarrow \overline{L^{\prime}}$ be the map given by Lemma 2.20 with $\phi$ being the inclusion

$$
V_{q}(\lambda) \rightarrow V_{q}\left(\omega_{i_{1}, a_{i_{1}}, \lambda\left(h_{i_{1}}\right)}\right) \otimes \cdots \otimes V_{q}\left(\omega_{i_{n}, a_{i_{n}}, \lambda\left(h_{i_{n}}\right)}\right),
$$

after identifying $V_{q}(\lambda)$ with $U_{q}(\tilde{\mathfrak{g}}) v$. It follows that $\tau_{a}^{*}(\bar{\phi}): L(\lambda) \rightarrow M\left(\lambda\left(h_{i_{1}}\right) \omega_{i_{1}}\right) \otimes$ $\cdots \otimes M\left(\lambda\left(h_{i_{n}}\right) \omega_{i_{n}}\right)$ is a $\mathfrak{g}[t]$-module map whose image is $T(\lambda)$.

4.5. A smaller set of relations for $M(\lambda)$. In this subsection, $\mathfrak{g}$ is orthogonal. Let

$$
R_{1}^{+}=\left\{\alpha \in R^{+}: \alpha=\sum_{i \in I} n_{i} \alpha_{i} \text { with } n_{i} \leq 1 \text { for all } i \in I\right\} .
$$


Proposition 4.6. For every $\lambda \in P^{+}$, the module $M(\lambda)$ is isomorphic to the $\mathfrak{g}[t]-$ module $N(\lambda)$ generated by a vector $v$ satisfying, for all $\alpha \in R_{1}^{+}$,

$$
h_{i} v=\lambda\left(h_{i}\right) v \quad \text { and } \quad \mathfrak{n}^{+}[t] v=\mathfrak{h} \otimes t \mathbb{C}[t] v=\left(x_{\alpha_{i}}^{-}\right)^{\lambda\left(h_{i}\right)+1} v=x_{\alpha, 1}^{-} v=0 .
$$

Proof. Since every admissible $J \subseteq I$ is of type $A$, it follows that $N(\lambda)$ is a quotient of $A(\lambda)$ and hence a finite-dimensional restricted $\mathfrak{g}[t]$-module. Moreover, it is easy to see that $M(\lambda)$ is a quotient of $N(\lambda)$. For the converse, set $\alpha_{i, j}=\sum_{k=i}^{j} \alpha_{k}$ for all $i, j \in I$ and $i \leq j$ if $\mathfrak{g}$ is of type $B$, and for all $i \leq j<n$ if $\mathfrak{g}$ is of type $D$. If $\mathfrak{g}$ is of type $D$, set also $\alpha_{i, n}=\alpha_{i, n-2}+\alpha_{n}$ for $i<n-1$ or $i=n$ and $\vartheta_{i}=\alpha_{i, n-1}+\alpha_{n}$ for $i \leq n-2$. Furthermore, given $i \leq j<n$ (or $j<n-2$ if $\mathfrak{g}$ is of type $D$ ) set

$$
\theta_{i, j}= \begin{cases}\alpha_{i, n}+\alpha_{j+1, n} & \text { if } \mathfrak{g} \text { is of type } B_{n}, \\ \alpha_{i, n-1}+\alpha_{j+1, n} & \text { if } \mathfrak{g} \text { is of type } D_{n} .\end{cases}
$$

Then $R_{1}^{+}=\left\{\alpha_{i, j}: i, j \in I\right\}$ (or $R_{1}^{+}=\left\{\alpha_{i, j}: i, j \in I\right\} \cup\left\{\vartheta_{i}: i \leq n-2\right\}$ if $\mathfrak{g}$ is of type $D)$ and $R^{+}=R_{1}^{+} \cup\left\{\theta_{i, j}: i, j \in I\right\}$.

Denote by $v_{i, m}$ the image of 1 in $M\left(m \omega_{i}\right)$ for $i \in I$ and $m \in \mathbb{Z}_{\geq 0}$. Since $R^{+}(i, m, 0)=R^{+}$if $m=0$, we shall assume $m>0$. Moreover, since we already know that Proposition 4.6 holds when $\lambda$ is a multiple of a fundamental weight, we assume from now on that $\lambda \in P^{+}$is not a multiple of a fundamental weight. From here we split the proof that $N(\lambda)$ is a quotient of $M(\lambda)$ in separate cases according to the type of $\mathfrak{g}$.

Case: $\mathfrak{g}$ is of type B. It follows from [Chari 2001; CM 2006] that

$$
\begin{array}{ll}
R^{+}(i, m, 0)=\left\{\alpha_{j, k}: i<j \text { or } k<i\right\} \cup\left\{\theta_{j, k}: i<j\right\} & \text { and } \\
R^{+}(n, 1,1)=R^{+}(i, m, 2)=R^{+} & \text {for all } i \in I \text { and } m>0
\end{array}
$$

and

$$
R^{+}(i, m, 1)=R^{+}(i, m, 0) \cup\left\{\alpha_{j, k}: j \leq i \leq k\right\} \cup\left\{\theta_{j, k}: i \leq k\right\} \quad \text { if }(i, m) \neq(n, 1) .
$$

Set

$$
i_{\lambda}= \begin{cases}\min \left\{i: \lambda\left(h_{j}\right)=0 \text { for all } j>i\right\} & \text { if } \lambda\left(h_{n}\right) \neq 1, \\ \min \left\{i: \lambda\left(h_{j}\right)=0 \text { for all } i<j<n\right\} & \text { otherwise }\end{cases}
$$

It follows from the above that

$$
R^{+}(\lambda, 1)=R^{+}\left(i_{\lambda}, \lambda\left(h_{i_{\lambda}}\right), 1\right)=R^{+} \backslash\left\{\theta_{j, k}: k<i_{\lambda}\right\} .
$$

Proposition 4.6 follows immediately in the case $\lambda\left(h_{n}\right)>1$.

To complete the proof of Proposition 4.6, assume first that $\lambda\left(h_{n}\right)=0$ and notice that $x_{\alpha_{i, j}}^{-} v=x_{\theta_{i, j}}^{-} v=0$ if $i>i_{\lambda}$. It follows that

$$
x_{\theta_{i, j}, r}^{-} v=\left[x_{\alpha_{i, n}, r}^{-}, x_{\alpha_{j, n}}^{-}\right] v=0 \quad \text { for all } i, j \in I, j>i_{\lambda} \text { and } r \in \mathbb{Z}_{>0} .
$$


Also, if $r>1$ and $j \leq i_{\lambda}$, then $x_{\theta_{i, j}, r}^{-} v=\left[x_{\alpha_{i, n}, r-1}^{-}, x_{\alpha_{j+1, n}, 1}^{-}\right] v=0$. This completes the proof in this case.

If $\lambda\left(h_{n}\right)=1$, then $x_{\alpha_{i, j}}^{-} v=0$ if $i_{\lambda}<i \leq j<n$. Therefore, to conclude the proof, it suffices to show that $x_{\theta_{i, n-1}, 1}^{-} v=0$ for all $i>i_{\lambda}$. We prove this inductively on $n-i$. In fact, it follows from the PBW theorem that

$$
N(\lambda)[1] \subseteq \sum_{i \leq j<n} U(\mathfrak{g}) x_{\theta_{i, j}, 1}^{-} v .
$$

In particular, the set of weights of $N(\lambda)$ [1] is contained in $S-Q^{+}$, where $S=$ $\left\{\lambda-\theta_{i, j}: i \leq j<n\right\}$. It is easy to see that $\lambda-\theta_{n-1, n-1}$ is a maximal element of $S$. Hence, if $x_{\theta_{n-1, n-1}, 1} v$ were nonzero, $V\left(\lambda-\theta_{n-1, n-1}\right)$ would be an irreducible constituent of $N(\lambda)[1]$. But the condition $\lambda\left(h_{n}\right)=1$ implies $\lambda-\theta_{n-1, n-1} \notin P^{+}$. Since $N(\lambda)$ is finite-dimensional, the inductive argument starts. Now suppose $x_{\theta_{i, n-1}, 1}^{-} v=0$ for all $i \geq j$ for some $j \leq n-1$ and observe that $\lambda-\theta_{j-1, n-1}$ is a maximal element of $S \backslash\left\{\lambda-\theta_{i, n-1}: i \geq j\right\}$. Once more $\lambda-\theta_{j-1, n-1} \notin P^{+}$, and we conclude the inductive argument as before.

Case: $\mathfrak{g}$ is of type $D$. In this case we have

$$
\begin{aligned}
& R^{+}(i, m, 0)=\left\{\alpha_{j, k}: i<j \text { or } k<i\right\} \cup\left\{\vartheta_{j}, \theta_{j, k}: i<j\right\} \text { if } i \neq n, n-1 \text {, } \\
& R^{+}(i, m, 0)=\left\{\alpha_{j, k}: k<n-1 \text { or } k=i^{\prime}\right\} \quad \text { if }\left\{i, i^{\prime}\right\}=\{n, n-1\}, \\
& R^{+}(1, m, 1)=R^{+}(n-1, m, 1)=R^{+}(n, m, 1)=R^{+}(i, m, 2)=R^{+} \text {for all } i \in I \text {, } \\
& R^{+}(i, m, 1)=R^{+}(i, m, 0) \cup\left\{\alpha_{j, k}: j \leq i \leq k\right\} \cup\left\{\theta_{j, k}: i \leq k\right\} \quad \text { if } i \notin\{1, n-1, n\} \text {. }
\end{aligned}
$$

In particular,

$$
R^{+}(\lambda, 1)=R^{+} \quad \text { if } \lambda\left(h_{i}\right)=0 \text { for all } i \notin\{1, n-1, n\},
$$

and hence $M(\lambda)$ is an irreducible $\mathfrak{g}$-module. Set

$i_{\lambda}= \begin{cases}1 & \text { if } \lambda\left(h_{i}\right)=0 \text { for all } i \notin\{1, n-1, n\}, \\ \min \left\{i: \lambda\left(h_{j}\right)=0 \text { for } i<j<n-1\right\} & \text { otherwise. }\end{cases}$

It follows that $R^{+}(\lambda, 1)=R^{+}\left(i_{\lambda}, \lambda\left(h_{i_{\lambda}}\right), 1\right)=R^{+} \backslash\left\{\theta_{j, k}: k<i_{\lambda}\right\}$. We are left to show that $x_{\theta_{i, j}, 1}^{-} v=0$ if $j>i_{\lambda}$. But this is clear since $x_{\alpha_{j, n-2}}^{-} v=0$ if $i_{\lambda}<j$ and $x_{\theta_{i, j}, 1}^{-}=\left[x_{\alpha_{j, n-2}}^{-}, x_{\vartheta_{i}, 1}^{-}\right]$.

The following corollary is now immediate and proves the first isomorphism of Conjecture 3.20 in some very particular cases.

Corollary 4.7. Suppose $\lambda \in P^{+}$is such that

(a) $\lambda\left(h_{i}\right)=0$ for all $1<i<n$ and $\lambda\left(h_{n}\right) \leq 1$ if $\mathfrak{g}$ is of type $B$, and

(b) $\lambda\left(h_{i}\right)=0$ for all $i \notin\{1, n-1, n\}$ if $\mathfrak{g}$ is of type $D$.

Then, $M(\lambda)$ is irreducible as a $\mathfrak{g}$-module. In particular, $M(\lambda) \cong T(\lambda)$. 
4.8. The l-characters. Let $\mathbb{Z}\left[\mathscr{P}_{q}\right]$ be the integral group ring over $\mathscr{P}_{q}$. We define the $\ell$-character of $V \in \widetilde{\mathscr{C}}_{q}$ to be the element $\operatorname{ch}_{\ell}(V)=\sum_{\mu \in \mathscr{P}_{q}} \operatorname{dim}\left(V_{\mu}\right) \mu$. of $\mathbb{Z}\left[\mathscr{P}_{q}\right]$. The $\ell$-characters are better known as $q$-characters, since this was the name used when they were first defined in [Frenkel and Reshetikhin 1999]. We prefer " $\ell$ characters" for two reasons: they record information about the dimension of the $\ell$-weight spaces of $V$ (which are not known as $q$-weight spaces), and the definition makes sense in the classical context as well. However, due to Theorem 2.12, the study of $\ell$-characters in the classical case easily reduces to the study of characters, and so $\ell$-characters are interesting only in the quantum case.

The proofs of the next four results can be found in [CM 2005; Frenkel and Mukhin 2001].

Proposition 4.9. Let $\mathfrak{g}=\mathfrak{s l}_{2}, a \in \mathbb{C}(q)^{\times}$, and $r \in \mathbb{Z}_{\geq 0}$. Then

$$
\operatorname{ch}_{\ell}\left(V_{q}\left(\boldsymbol{\omega}_{i, a, r}\right)\right)=\boldsymbol{\omega}_{i, a, r} \sum_{k=0}^{r}\left(\prod_{j=1}^{k} \boldsymbol{\omega}_{i, a q^{r-2 j, 2}}\right)^{-1}=\boldsymbol{\omega}_{i, a, r} \sum_{k=0}^{r}\left(\prod_{j=1}^{k} \boldsymbol{\alpha}_{i, a q^{r-2 j+1}}\right)^{-1} .
$$

Theorem 4.10. Let $V$ be a quotient of $W_{q}(\lambda)$ for some $\lambda \in \mathscr{P}_{q}^{+}$. If $V_{\mu} \neq 0$, then $\mu \leq \lambda$.

Proposition 4.11. Let $V \in \widetilde{\mathscr{C}}_{q}, v \in V_{\boldsymbol{\mu}} \backslash\{0\}$ for some $\boldsymbol{\mu} \in \mathscr{P}_{q}$, and suppose $i \in I$ is such that $x_{i, r}^{+} v=0$ for all $r \in \mathbb{Z}$. Then, $\boldsymbol{\mu}_{i}(u) \in \mathbb{C}(q)[u]$, and if $\boldsymbol{\mu}_{i}(u)=$ $\prod_{k=1}^{m} f_{i, a_{k}, r_{k}}(u)$ as in (1-14), then

$$
x_{i}^{-} v \in \sum_{k=1}^{m} \sum_{j=1}^{r_{k}} V_{\boldsymbol{\mu}\left(\boldsymbol{\alpha}_{i, a_{k} q_{i} r_{k}+1-2 j}\right)^{-1}}
$$

Moreover, $\operatorname{dim}\left(V_{\boldsymbol{\mu}\left(\boldsymbol{\alpha}_{i, a_{k}} q^{r-1}\right)^{-1}}\right) \geq \#\left\{1 \leq l \leq k: a_{l}=a_{k}\right\}$.

Given $V \in \widetilde{\mathscr{C}}_{q}$, let $\mathrm{wt}_{\ell}(V)=\left\{\boldsymbol{\mu} \in \mathscr{P}_{q}: V_{\boldsymbol{\mu}} \neq 0\right\}$. A highest- $\ell$-weight module $V$ of highest $\ell$-weight $\lambda \in \mathscr{P}_{q}^{+}$is said to be special if $\mathrm{wt}_{\ell}(V) \cap \mathscr{P}_{q}^{+}=\{\lambda\}$.

Theorem 4.12. If $\lambda \in \mathscr{P}_{q}^{+}$is such that $V_{q}(\lambda)$ is special, then the output of the Frenkel-Mukhin algorithm with input $\lambda$ is $\operatorname{char}_{\ell}\left(V_{q}(\lambda)\right)$.

Theorem 4.13 [Hernandez 2007]. If $\mathfrak{g}$ is of type $A, B$, or $G$, then all minimal affinizations are special.

Let $\mathfrak{g}$ be of type $A, B$, or $G$, and let $\lambda \in P^{+}$. It follows from the above that if $V_{q}(\lambda)$ is a minimal affinization of $V_{q}(\lambda)$, then $\operatorname{char}_{\ell}\left(V_{q}(\lambda)\right)$ is given by the Frenkel-Mukhin algorithm. We will actually need only the following corollary of the algorithm. Let $V \in \widetilde{\mathscr{C}}_{q}$ and $v \in V_{\mu} \backslash\{0\}$ for some $\boldsymbol{\mu} \in \mathscr{P}_{q}$, and suppose $i \in I$ is such that $x_{i, r}^{+} v=0$ for all $r \in \mathbb{Z}$. Using Proposition 4.11, we can write 
$\boldsymbol{\mu}_{i}(u)=\prod_{k=1}^{m} f_{i, a_{k}, r_{k}}(u)$ as in (1-14). Then, the algorithm implies that (4-3) $\quad \boldsymbol{\mu} \boldsymbol{\alpha}_{i, b}^{-1} \in \mathrm{wt}_{\ell}\left(V_{q}(\lambda)\right) \quad$ if and only if $b=a_{k} q_{i}^{r_{k}-1}$ for some $k=1, \ldots, m$.

The next proposition will be crucial for the proof of Proposition 3.22.

Proposition 4.14. Suppose $\mathfrak{g}$ is of type A; let $\lambda \in P^{+}, \lambda=\prod_{i \in I} \boldsymbol{\omega}_{i, a_{i}, \lambda\left(h_{i}\right)}$, $\boldsymbol{\mu} \in \mathrm{wt}_{\ell}\left(V_{q}(\lambda)\right)$, and $\lambda \boldsymbol{\mu}^{-1}=\boldsymbol{\alpha}_{j, b_{j}} \boldsymbol{\alpha}_{j+1, b_{j+1}} \cdots \boldsymbol{\alpha}_{k, b_{k}}$ for some $j \leq k$ and some $a_{i}, b_{l} \in \mathbb{C}(q)^{\times}$and $i \in I, l=j, \ldots, k$.

(a) If $a_{i+1} / a_{i}=q^{\lambda\left(h_{i}\right)+\lambda\left(h_{i+1}\right)+1}$ for all $i<n$, then $b_{k}=a_{k} q^{\lambda\left(h_{k}\right)-1}$.

(b) If $a_{i+1} / a_{i}=q^{-\left(\lambda\left(h_{i}\right)+\lambda\left(h_{i+1}\right)+1\right)}$ for all $i<n$, then $b_{j}=a_{j} q^{\lambda\left(h_{j}\right)-1}$.

Proof. This is straightforward using induction on $k-j$ together with (4-3).

\subsection{Quantized relations.}

Proof of Proposition 3.22. We now assume that $\mathfrak{g}$ is orthogonal. To make the notation more uniform, we assume for the rest of the proof that $\mathfrak{g}$ is of type $B_{n}$ or $D_{n+1}$ for $n \geq 2$. Before we begin, let us record the following corollary of Proposition 3.22 and Corollary 4.7.

Corollary 4.16. If $\lambda$ satisfies the conditions of Proposition 3.22 and Corollary 4.7, then Conjecture 3.20 holds. In particular, if $V_{q}(\lambda)$ is a minimal affinization of $V_{q}(\lambda)$, then $V_{q}(\lambda) \cong V_{q}(\lambda)$ as a $U_{q}(\mathfrak{g})$-module.

If $\lambda$ is supported on an admissible subdiagram, Proposition 3.22 easily follows from Propositions 3.13 and 4.6. In particular, we can henceforth assume that the support of $\lambda$ contains a spin node and that there exists $i<n$ such that $\lambda\left(h_{i}\right) \neq 0$. If $\mathfrak{g}$ is of type $D$, we will prove Proposition 3.22 in the case $\lambda\left(h_{n+1}\right)=0$; the other cases are proved similarly. Set

$$
i_{\lambda}=\min \left\{i: \lambda\left(h_{j}\right)=0 \text { for all } i<j<n\right\} .
$$

This definition of $i_{\lambda}$ does not coincide with the one given in Section 4.5 for $\mathfrak{g}$ of type $B$ and $\lambda\left(h_{n}\right)>1$.

From now on we assume that $\lambda \in \mathscr{P}_{\mathrm{A}}^{++}$is such that $V=V_{q}(\lambda)$ is a minimal affinization of $V_{q}(\lambda)$ and $a \in \mathbb{C}$ is such that $\bar{\lambda}=\boldsymbol{\omega}_{\lambda, a}$. We also fix a highest$\ell$-weight $v$ vector of $V$ and $a_{i} \in \mathbb{A}^{\times}$for $i \in I$, such that $\lambda=\prod_{i \in I} \omega_{i, a_{i}, \lambda\left(h_{i}\right)}$.

Let $v^{\prime}$ be the image of $v$ in $L(\lambda)$. It again follows from Proposition 3.13 that $x_{\alpha_{i, j}, 1}^{-} v^{\prime}=0$ if $i=j$ or if $j<n$. If $\mathfrak{g}$ is of type $B$, this implies $x_{\alpha_{i, n}, 1}^{-} v^{\prime}=0$ if $i>i_{\lambda}$. If $\mathfrak{g}$ is of type $D$ and $i_{\lambda}<i<n$, it follows that $x_{\alpha_{i, n}, 1}^{-} v^{\prime}=x_{\alpha_{i, n+1}, 1}^{-} v^{\prime}=x_{\vartheta_{i}, 1}^{-} v^{\prime}=0$. We claim that it remains to show that $x_{\alpha_{i, n}, 1}^{-} v^{\prime}=0$ for $i \leq i_{\lambda}$. In fact, if $\mathfrak{g}$ is of type $B$ this is clear from Proposition 4.6. If $\mathfrak{g}$ is of type $D$, it follows that $x_{\vartheta_{i}, 1}^{-} v^{\prime}=\left[x_{\alpha_{n+1}}^{-}, x_{\alpha_{i, n}, 1}^{-}\right] v^{\prime}=0$ provided $x_{\alpha_{i, n}, 1}^{-} v^{\prime}=0$. 
Denote by $\bar{v}$ the image of $v$ in $\bar{V}$. It suffices to show that $x_{\alpha_{i, n}, 1}^{-} \bar{v}=a x_{\alpha_{i, n}}^{-} \bar{v}$ for all $i \leq i_{\lambda}$. Consider the elements

$$
X_{\alpha_{i, j}, r}^{-}=\left[x_{j, r}^{-},\left[x_{j-1}^{-}, \ldots\left[x_{i+1}^{-}, x_{i}^{-}\right] \ldots\right]\right] \quad \text { and } \quad k_{i, j}=\prod_{l=i}^{j} k_{l}
$$

for $i \leq j \leq n$ and $r \in \mathbb{Z}_{\geq 0}$. Set $X_{\alpha_{i, j}}^{-}:=X_{\alpha_{i, j}, 0}^{-}$, and note that $X_{\alpha_{i, j}, r}^{-}=\left[x_{j, r}^{-}, X_{\alpha_{i, j-1}}^{-}\right]$ if $i<j$. Clearly

$$
X_{\alpha_{i, j}, r}^{-} \in U_{\mathcal{A}}\left(\tilde{\mathfrak{n}}^{-}\right) \quad \text { and } \quad \overline{X_{\alpha_{i, j}, r}^{-}}=x_{\alpha_{i, j}, r}^{-} \text {. }
$$

Lemma 4.17. Suppose $V \in \mathscr{C}_{q}, \quad \mu \in P, w \in V_{\mu} \backslash\{0\}$, and $i \in I$ are such that $\mu\left(h_{l}\right)=0$ and $x_{l}^{+} w=0$ for $l>i$. Then $X_{\alpha_{i, j}}^{-} w=x_{j}^{-} x_{j-1}^{-} \cdots x_{i}^{-} w$.

Proof. This is a straightforward computation using the commutation relations $\left[x_{l, s}^{-}, x_{l^{\prime}, s^{\prime}}^{-}\right]=0$ for $l, l^{\prime}$ such that $c_{l, l^{\prime}}=0$ and $x_{l}^{-} w=0$ if $l>i$.

Lemma 4.18. Suppose $w$ is a highest- $\ell$-weight vector of $V_{q}\left(\omega_{i, a_{i}, m}\right)$ for some $i \in I$, and some $m \in \mathbb{Z}_{\geq 0}$. Then $x_{i, 1}^{-} w=a_{i} q_{i}^{m} x_{i}^{-} w$.

Proof. Use the relation $\left[h_{i, 1}, x_{i}^{-}\right]=-[2]_{q_{i}} x_{i, 1}^{-}$and Proposition 4.9.

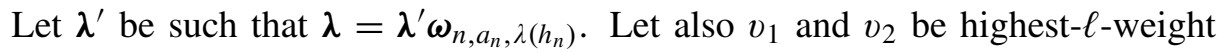
vectors of $V_{q}\left(\lambda^{\prime}\right)$ and $V_{q}\left(\omega_{n, a_{n}, \lambda\left(h_{n}\right)}\right)$. By Proposition 4.3 and Corollary 4.4, either

$$
\begin{aligned}
& V_{q}(\lambda) \cong U_{q}(\tilde{\mathfrak{g}})\left(v_{1} \otimes v_{2}\right) \subseteq V_{q}\left(\boldsymbol{\lambda}^{\prime}\right) \otimes V_{q}\left(\boldsymbol{\omega}_{n, a_{n}, \lambda\left(h_{n}\right)}\right) \text { or } \\
& V_{q}(\boldsymbol{\lambda}) \cong U_{q}(\tilde{\mathfrak{g}})\left(v_{2} \otimes v_{1}\right) \subseteq V_{q}\left(\boldsymbol{\omega}_{n, a_{n}, \lambda\left(h_{n}\right)}\right) \otimes V_{q}\left(\boldsymbol{\lambda}^{\prime}\right) .
\end{aligned}
$$

We assume we are in the first case (the latter is proved similarly using part (b) of Proposition 4.14 instead of part (a)). In particular, by Theorem 3.2, we must have

$$
a_{i+1} / a_{i}=q^{d_{i} \lambda\left(h_{i}\right)+d_{i+1} \lambda\left(h_{i+1}\right)+r^{\vee}} \quad \text { for all } i<n .
$$

By Lemmas 1.5 and 1.6, modulo elements of the form $x\left(v_{1} \otimes v_{2}\right)$ with $x \in$ $U_{\mathrm{A}}(\tilde{\mathfrak{g}}) \otimes U_{\mathrm{A}}(\tilde{\mathfrak{g}})$ such that $\bar{x}=0$, we have

$$
\begin{aligned}
X_{\alpha_{i, n}}^{-}\left(v_{1} \otimes v_{2}\right)= & x_{n}^{-} X_{\alpha_{i, n-1}}^{-}\left(v_{1} \otimes v_{2}\right)-X_{\alpha_{i, n-1}}^{-} x_{n}^{-}\left(v_{1} \otimes v_{2}\right) \\
= & x_{n}^{-}\left(\left(X_{\alpha_{i, n}}^{-} v_{1}\right) \otimes v_{2}\right)-X_{\alpha_{i, n-1}}^{-}\left(v_{1} \otimes\left(x_{n}^{-} v_{2}\right)\right) \\
= & \left(x_{n}^{-} X_{\alpha_{i, n-1}}^{-} v_{1}\right) \otimes\left(q^{-\lambda\left(h_{n}\right)} v_{2}\right)+\left(X_{\alpha_{i, n-1}}^{-} v_{1}\right) \otimes\left(x_{n}^{-} v_{2}\right) \\
& \quad-\left(X_{\alpha_{i, n-1}}^{-} v_{1}\right) \otimes\left(k_{i, n-1}^{-1} x_{n}^{-} v_{2}\right)-v_{1} \otimes\left(X_{\alpha_{i, n-1}}^{-} x_{n}^{-} v_{2}\right) \\
= & q^{-\lambda\left(h_{n}\right)}\left(x_{n}^{-} X_{\alpha_{i, n-1}}^{-} v_{1}\right) \otimes v_{2}+\left(1-q^{-2}\right)\left(X_{\alpha_{i, n-1}}^{-} v_{1}\right) \otimes\left(x_{n}^{-} v_{2}\right)
\end{aligned}
$$


On the other hand,

$$
\begin{aligned}
X_{\alpha_{i, n}, 1}^{-}\left(v_{1} \otimes v_{2}\right)= & x_{n, 1}^{-} X_{\alpha_{i, n-1}}^{-}\left(v_{1} \otimes v_{2}\right)-X_{\alpha_{i, n-1}}^{-} x_{n, 1}^{-}\left(v_{1} \otimes v_{2}\right) \\
= & x_{n, 1}^{-}\left(\left(X_{\alpha_{i, n}}^{-} v_{1}\right) \otimes v_{2}\right)-X_{\alpha_{i, n-1}}^{-}\left(v_{1} \otimes\left(x_{n, 1}^{-} v_{2}\right)\right) \\
= & \left(x_{n, 1}^{-} X_{\alpha_{i, n-1}}^{-} v_{1}\right) \otimes\left(q^{\lambda\left(h_{n}\right)} v_{2}\right)+\left(X_{\alpha_{i, n-1}}^{-} v_{1}\right) \otimes\left(x_{n, 1}^{-} v_{2}\right) \\
& \quad-\left(X_{\alpha_{i, n-1}}^{-} v_{1}\right) \otimes\left(k_{i, n-1}^{-1} x_{n, 1}^{-} v_{2}\right)-v_{1} \otimes\left(X_{\alpha_{i, n-1}}^{-} x_{n, 1}^{-} v_{2}\right) \\
= & q^{\lambda\left(h_{n}\right)}\left(x_{n, 1}^{-} X_{\alpha_{i, n-1}}^{-} v_{1}\right) \otimes v_{2}+\left(1-q^{-2}\right)\left(X_{\alpha_{i, n-1}}^{-} v_{1}\right) \otimes\left(x_{n, 1}^{-} v_{2}\right)
\end{aligned}
$$

Using Lemma 4.18 we get

$$
\begin{aligned}
& X_{\alpha_{i, n}, 1}^{-}\left(v_{1} \otimes v_{2}\right) \\
& =q^{\lambda\left(h_{n}\right)}\left(x_{n, 1}^{-} X_{\alpha_{i, n-1}}^{-} v_{1}\right) \otimes v_{2} \\
& +a_{n} q^{\lambda\left(h_{n}\right)}\left(\left(1-q^{-2}\right)\left(X_{\alpha_{i, n-1}}^{-} v_{1}\right) \otimes\left(x_{n}^{-} v_{2}\right)-v_{1} \otimes\left(X_{\alpha_{i, n-1}}^{-} x_{n}^{-} v_{2}\right)\right) \\
& =a_{n} q^{\lambda\left(h_{n}\right)} X_{\alpha_{i, n}}^{-}\left(v_{1} \otimes v_{2}\right)+q^{\lambda\left(h_{n}\right)}\left(x_{n, 1}^{-} X_{\alpha_{i, n-1}}^{-} v_{1}\right) \otimes v_{2}-a_{n}\left(x_{n}^{-} X_{\alpha_{i, n-1}}^{-} v_{1}\right) \otimes v_{2} \text {. }
\end{aligned}
$$

Hence, it suffices to show that

$$
q^{\lambda\left(h_{n}\right)}\left(x_{n, 1}^{-} X_{\alpha_{i, n-1}}^{-} v_{1}\right) \otimes v_{2}=a_{n}\left(x_{n}^{-} X_{\alpha_{i, n-1}}^{-} v_{1}\right) \otimes v_{2} .
$$

If $i>i_{\lambda}$, both sides of the above equality vanish. If $i \leq i_{\lambda}$, we proceed as follows. Notice that $x_{n, r}^{+} X_{\alpha_{i, n-1}}^{-} v_{1}=0$ for all $r \in \mathbb{Z}$, and let $W$ be the $U_{q}\left(\tilde{\mathfrak{g}}_{n}\right)$-submodule of $V_{q}\left(\lambda^{\prime}\right)$ generated by $X_{\alpha_{i, n-1}}^{-} v_{1}$. Then, by Proposition 4.14(a), the highest- $\ell$-weight of $W$ is $\omega_{n, a_{n-1}} q^{r^{\vee} \lambda\left(h_{n-1}\right)}, r^{\vee}$. Moreover, by (4-3), $W$ is a minimal affinization. Hence, by Lemma 4.18 ,

$$
x_{n, 1}^{-} X_{\alpha_{i, n-1}}^{-} v_{1}=a_{n-1} q^{r^{\vee}\left(\lambda\left(h_{n-1}\right)+1\right)} x_{n}^{-} X_{\alpha_{i, n-1}}^{-} v_{1} .
$$

This and (4-5) imply (4-6).

\section{Graded characters of restricted limits of minimal affinizations}

5.1. Preliminaries. Although Theorem 3.2 tells which objects of $\widetilde{\mathscr{C}}_{q}$ correspond to minimal affinizations, it does not say anything about their $U_{q}(\mathfrak{g})$-structure, unless $\mathfrak{g}$ is of type $A$. In some few cases this is known; see [Chari 1995; CM 2006; 2007]. In principle, the $U_{q}(\mathfrak{g})$-structure can be read off the $\ell$-character. In practice, this is not so easy to do, even in the situations that the Frenkel-Mukhin algorithm does produced the $\ell$-character. We will now apply the techniques of [CM 2006; 2007] to prove Proposition 3.24 and, hence, Conjecture 3.20 in those cases. As a byproduct, we obtain closed formulas for the character of the minimal affinizations if $\lambda$ is as 
in Proposition 3.24. We shall also prove an analogue of Conjecture 3.20 in the case of multiple minimal affinizations for $\mathfrak{g}$ of type $D_{4}$.

Lemma 5.2 [CM 2007, Section 1.5]. Consider the three-dimensional Heisenberg algebra $\mathfrak{H}$ spanned by elements $x, y$ and $z$, where $z$ is central and $[x, y]=z$. Suppose that $V$ is a representation of $\mathfrak{H}$, and let $0 \neq v \in V$ be such that $x^{r} v=0$. Then for all $k, s \in \mathbb{Z}_{\geq 0}$, the element $y^{k} z^{s} v$ is in the span of elements of the form $x^{a} y^{b} z^{c} v$ with $0 \leq c<r, a+c=s$, and $b+c=k+s$.

Let $V$ be a finite-dimensional $\mathfrak{g}$-module, and let $\lambda \in P^{+}$. Denote by $m_{\lambda}(V)$ the multiplicity of the irreducible module $V(\lambda)$ as an irreducible constituent of $V$. Set $d_{i}^{\prime}=d_{i} / r^{\vee}$. Hence, if $\mathfrak{g}$ is simply laced, $d_{i}^{\prime}=d_{i}=1$. If $\mathfrak{g}$ is of type $B, d_{i}^{\prime}=1$ if $i<n$ and $d_{n}^{\prime}=1 / 2$. The symbol $[m]$ means the largest integer not greater than $m$.

5.3. Type B. Given $i \in I$ and $m \in \mathbb{Z}_{\geq 0}$, let $v_{i, m}$ be the image of 1 in $M\left(m \omega_{i}\right)$. The following was proved in.

Lemma 5.4 [Chari 2001; CM 2006]. (a) $M\left(m \omega_{1}\right) \cong V\left(m \omega_{1}\right)$.

(b) $M\left(m \omega_{2}\right)[l]= \begin{cases}0 & \text { if } l>\left[d_{2}^{\prime} m\right], \\ U\left(\mathfrak{n}^{-}\right)\left(x_{\theta_{1,1}, 1}^{-}\right)^{l} v_{2, m} \cong V\left(m \omega_{2}-l \theta_{1,1}\right) & \text { if } 0 \leq l \leq\left[d_{2}^{\prime} m\right] .\end{cases}$
(c) $M\left(m \omega_{3}\right)[l]= \begin{cases}0 & \text { if } l>\left[d_{3}^{\prime} m\right], \\ U\left(\mathfrak{n}^{-}\right)\left(x_{\theta_{2,2}, 1}^{-}\right)^{l} v_{3, m} \cong V\left(m \omega_{3}-l \theta_{2,2}\right) & \text { if } 0 \leq l \leq\left[d_{3}^{\prime} m\right] .\end{cases}$

Also, $\left(x_{\theta_{1,2}, 1}^{-}\right)^{r_{1,2}}\left(x_{\theta_{2,2}, 1}^{-}\right)^{r_{2,2}} v_{3, m}$ is a multiple of $\left(x_{\alpha_{1}}^{-}\right)^{r_{1,2}}\left(x_{\theta_{2,2}, 1}^{-}\right)^{r_{1,2}+r_{2,2}} v_{3, m}$.

The "also" part of this lemma can also be proved using Lemma 5.2.

Proposition 5.5. Suppose $\lambda \in P^{+}$is such that $\lambda\left(h_{i}\right)=0$ for $i>2$. Then,

$$
M(\lambda)[k] \cong \begin{cases}V\left(\lambda-k \theta_{1,1}\right) & \text { if } 0 \leq k \leq\left[d_{2}^{\prime} \lambda\left(h_{2}\right)\right], \\ 0 & \text { otherwise. }\end{cases}
$$

Also, $M(\lambda) \cong T(\lambda)$.

Proof. Let $v$ be the image of 1 in $M(\lambda)$. Equation (4-1) gives $R^{+}(\lambda, 1) \supseteq R^{+} \backslash\left\{\theta_{1,1}\right\}$. Together with the PBW Theorem, this implies that

$$
M(\lambda)=\bigoplus_{k \geq 0} U\left(\mathfrak{n}^{-}\right)\left(x_{\theta_{1,1}, 1}^{-}\right)^{k} v .
$$

It follows from Lemma 2.3 that $m_{\mu}(M(\lambda)) \leq 1$ for every $\mu$ and that $m_{\mu}(M(\lambda))$ may be nonzero only when $\mu=\lambda-k \theta_{1,1}$ for some $k \in \mathbb{Z}_{\geq 0}$. Since $\theta_{1,1}=\left(d_{2}^{\prime}\right)^{-1} \omega_{2}$, $\mu-k \theta_{1,1} \in P^{+}$if and only if $k \leq\left[d_{2}^{\prime} \lambda\left(h_{2}\right)\right]$.

Let $v_{i}$ be a nonzero element in the top weight space of $M\left(\lambda\left(h_{i}\right) \omega_{i}\right)$ for $i=1,2$. Then, by [CM 2006], $\left(x_{\theta_{1,1}, 1}^{-}\right)^{k} v_{2}$ is the highest-weight vector of the irreducible $\mathfrak{g}$-submodule of $M\left(\lambda\left(h_{2}\right) \omega_{2}\right)[k]$ for $k=0, \ldots,\left[d_{2}^{\prime} \lambda\left(h_{2}\right)\right]$, while $M\left(\lambda\left(h_{1}\right) \omega_{1}\right)$ is an 
irreducible $\mathfrak{g}$-module itself. Therefore, $\left(x_{\theta_{1,1}, 1}^{-}\right)^{k}\left(v_{1} \otimes v_{2}\right)=v_{1} \otimes\left(x_{\theta_{1,1}, 1}^{-}\right)^{k} v_{2} \neq 0$, proving that $T(\lambda)[k] \neq 0$. Hence, $T(\lambda)[k] \cong M(\lambda)[k]$ and we are done.

Remark. This proposition reproves one of the main results of [Chari 1995] using a different method.

Now assume $n \geq 3$ and suppose $\lambda \in P^{+}$is such that $\lambda\left(h_{i}\right)=0$ for $i>3$. In this case, Equation (4-1) implies

$$
R^{+}(\lambda, 1) \supseteq R^{+} \backslash\left\{\theta_{2,2}, \theta_{1,2}, \theta_{1,1}\right\} .
$$

Observe that $\theta_{1,1}=\omega_{2}, \theta_{1,2}=\omega_{1}-\omega_{2}+\left(d_{3}^{\prime}\right)^{-1} \omega_{3}$ and $\theta_{2,2}=\left(d_{3}^{\prime}\right)^{-1} \omega_{3}-\omega_{1}$. In particular, $\left\{\theta_{2,2}, \theta_{1,2}, \theta_{1,1}\right\}$ is a linearly independent subset of $\mathfrak{h}^{*}$. Let $\mathbf{e}_{j}$ for $j \in \mathbb{Z}_{\geq 0}$ be the standard basis of $\mathbb{Z}_{\geq 0}^{3}$, set

$$
\mathscr{A}_{3}(\lambda)=\left\{\boldsymbol{r}=\left(r_{1}, r_{2}, r_{3}\right) \in \mathbb{Z}_{\geq 0}^{3}: r_{3} \leq \lambda\left(h_{2}\right), r_{2} \leq \lambda\left(h_{1}\right), r_{1}+r_{2} \leq\left[d_{3}^{\prime} \lambda\left(h_{3}\right)\right]\right\}
$$

and, given $r \in \mathbb{Z}_{\geq 0}^{3}$, define

$$
\mathbf{y}_{\boldsymbol{r}}=\left(x_{\theta_{2,2}, 1}^{-}\right)^{r_{1}}\left(x_{\theta_{1,2}, 1}^{-}\right)^{r_{2}}\left(x_{\theta_{1,1}, 1}^{-}\right)^{r_{3}} .
$$

Notice that the elements $x_{\theta_{2,2}, 1}^{-}, x_{\theta_{1,2}, 1}^{-}, x_{\theta_{1,1}, 1}^{-}$commute among themselves.

Lemma 5.6. Let $v$ be the image of 1 in $M(\lambda)$. For every $s \in \mathbb{Z}_{\geq 0}^{3}$,

$$
\mathbf{y}_{\boldsymbol{s}} v \in \sum_{\boldsymbol{r}} U\left(\mathfrak{n}^{-}\right) \mathbf{y}_{\boldsymbol{r}} v
$$

where the sum is over the elements $\boldsymbol{r} \in \mathbb{Z}_{\geq 0}^{3}$ such that $r_{3} \leq \lambda\left(h_{2}\right)$ and $r_{2} \leq \lambda\left(h_{1}\right)$.

Proof. By Lemma 5.2 with $x=x_{\alpha_{2}}^{-}, y=x_{\theta_{1,2}, 1}^{-}$, and $z=x_{\theta_{1,1}, 1}^{-}$, we have that $\mathbf{y}_{s} v$ is in the span of elements of the form $\left(x_{\alpha_{2}}^{-}\right)^{a} \mathbf{y}_{s^{\prime}} v$ with $a>0$ and $\mathbf{s}^{\prime}$ with $s_{3}^{\prime} \leq \lambda\left(h_{2}\right)$. Using Lemma 5.2 once more, this time with $x=x_{\alpha_{1}}^{-}, y=x_{\theta_{2,2}, 1}^{-}$, and $z=x_{\theta_{1,2}, 1}^{-}$, it follows that an element $\mathbf{y}_{\boldsymbol{s}^{\prime}} v$ with $\boldsymbol{s}^{\prime}$ as above belongs to the span of elements of the form $\left(x_{\alpha_{1}}^{-}\right)^{a} \mathbf{y}_{\boldsymbol{r}} v$ with $a>0$ and $\boldsymbol{r}$ as claimed.

Given $r \in \mathbb{Z}_{\geq 0}^{3}$, define

$$
\operatorname{wt}(\boldsymbol{r})=r_{1} \theta_{2,2}+r_{2} \theta_{1,2}+r_{3} \theta_{1,1} \quad \text { and } \operatorname{gr}(\boldsymbol{r})=r_{1}+r_{2}+r_{3} .
$$

Since $\left\{\theta_{2,2}, \theta_{1,2}, \theta_{1,1}\right\}$ is linearly independent, wt is an injective function.

Proposition 5.7. For every $\lambda \in P^{+}$as above, we have $M(\lambda) \cong T(\lambda)$ and

$$
M(\lambda)[l] \cong \bigoplus_{\boldsymbol{r} \in \mathscr{A}_{3}(\lambda): \operatorname{gr}(\boldsymbol{r})=l} V(\lambda-\mathrm{wt}(\boldsymbol{r}))
$$


Proof. Let $v$ be the image of 1 in $M(\lambda)$. Equation (5-1), together with the PBW theorem, implies that

$$
M(\lambda)=\sum_{r \in \mathbb{Z}_{\geq 0}^{3}} U\left(\mathfrak{n}^{-}\right) \mathbf{y}_{\boldsymbol{r}} v .
$$

Lemma 5.6 implies that the this sum can be restricted to $r$ such that $r_{3} \leq \lambda\left(h_{2}\right)$ and $r_{2} \leq \lambda\left(h_{1}\right)$. This, together with Lemma 2.3, implies that $m_{\mu}(M(\lambda)) \leq 1$ and equality may occur only if $\mu=\lambda-\operatorname{wt}(\boldsymbol{r})$ for some $\boldsymbol{r}$ as above. Also, $\operatorname{wt}(\boldsymbol{r}) \in P^{+}$ only if $r_{1}+r_{2} \leq\left[d_{3}^{\prime} \lambda\left(h_{3}\right)\right]$, and hence $\boldsymbol{r}$ must be in $\mathscr{A}_{3}(\lambda)$. It follows that $M(\lambda)[l]$ is a quotient of $\bigoplus_{r \in \mathscr{A}_{3}(\lambda): \operatorname{gr}(\boldsymbol{r})=l} V(\lambda-\operatorname{wt}(\boldsymbol{r}))$. To complete the proof, it suffices to show that $T(\lambda)[l]$ contains a submodule isomorphic to $V(\lambda-\operatorname{wt}(r))$ for every $\boldsymbol{r} \in \mathscr{A}_{3}(\lambda)$ such that $\operatorname{gr}(\boldsymbol{r})=l$.

Thus, let $v_{i}=v_{i, \lambda\left(h_{i}\right)}, i=1,2,3$, and let $v_{i}^{j}$ be the image of $v_{i}$ in $M\left(\lambda\left(h_{i}\right) \omega_{i}\right)(j)$ for $j \geq 0$. Then, if $\boldsymbol{r} \in \mathscr{A}_{3}(\lambda)$, Lemma 5.4 implies

$$
\begin{aligned}
\mathbf{y}_{\boldsymbol{r}}\left(v_{1} \otimes v_{2} \otimes v_{3}^{r_{1}+r_{2}}\right) & =v_{1} \otimes\left(x_{\theta_{1,1}, 1}^{-}\right)^{r_{3}} v_{2} \otimes\left(x_{\theta_{2,2}, 1}^{-}\right)^{r_{1}}\left(x_{\theta_{1,2}, 1}^{-}\right)^{r_{2}} v_{3}^{r_{1}+r_{2}} \\
& =v_{1} \otimes\left(x_{\theta_{1,1}, 1}^{-}\right)^{r_{3}} v_{2} \otimes\left(x_{\alpha_{1}}^{-}\right)^{r_{2}}\left(x_{\theta_{2,2}, 1}^{-}\right)^{r_{1}+r_{2}} v_{3}^{r_{1}+r_{2}} \neq 0 .
\end{aligned}
$$

Given $r \leq \lambda\left(h_{2}\right)$ and $s \leq\left[d_{3}^{\prime} \lambda\left(h_{3}\right)\right]$, let $T_{r, s}(\lambda)$ be the $\mathfrak{g}[t]$-submodule of

$$
M\left(\lambda\left(h_{1}\right) \omega_{1}\right) \otimes M\left(\lambda\left(h_{2}\right) \omega_{2}\right)(r) \otimes M\left(\lambda\left(h_{3}\right) \omega_{3}\right)(s)
$$

generated by $v_{r, s}:=v_{1} \otimes v_{2}^{r} \otimes v_{3}^{s}$. Clearly $T_{r, s}(\lambda)$ is a quotient of $T(\lambda)(r+s)$. Set $\boldsymbol{r}_{0}=(s, 0, r)$ and $\boldsymbol{r}_{j}=\boldsymbol{r}_{j-1}+\left(\mathbf{e}_{2}-\mathbf{e}_{1}\right)$ for $1 \leq j \leq s^{\prime}:=\min \left(\lambda\left(h_{1}\right), s\right)$. Notice that

$$
T_{r, s}(\lambda)[r+s]=\sum_{j=0}^{s^{\prime}} U\left(\mathfrak{n}^{-}\right) \mathbf{y}_{r_{j}} v_{r, s} \quad \text { and } \quad\left(\lambda-\operatorname{wt}\left(\boldsymbol{r}_{j}\right)\right)\left(h_{1}\right)=\lambda\left(h_{1}\right)+s .
$$

In particular, $\lambda-\operatorname{wt}\left(\boldsymbol{r}_{0}\right)$ is the unique maximal weight of $T_{r, s}(\lambda)[r+s]$. We prove inductively on $k=0,1, \ldots, s^{\prime}$ that

$$
\sum_{j=0}^{k} U\left(\mathfrak{n}^{-}\right) \mathbf{y}_{\boldsymbol{r}_{j}} v_{r, s} \cong \bigoplus_{j=0}^{k} V\left(\lambda-\mathrm{wt}\left(\boldsymbol{r}_{j}\right)\right)
$$

as a $\mathfrak{g}$-module. Since every $\boldsymbol{r} \in \mathscr{A}_{3}(\lambda)$ is of the form $\boldsymbol{r}_{j}$ for some $r, s, j$ as above, this completes the proof.

It is clear from Lemma 5.4 and (5-5) that $\mathfrak{n}^{+} \mathbf{y}_{r_{0}} v_{r, s}=0$ and hence generates a $\mathfrak{g}$-submodule isomorphic to $V\left(\lambda-\operatorname{wt}\left(\boldsymbol{r}_{0}\right)\right)$. In particular, we can assume $s^{\prime}>0$. Notice that the weight space of $V\left(\lambda-\operatorname{wt}\left(\boldsymbol{r}_{j}\right)\right)$ of weight $\lambda-\operatorname{wt}\left(\boldsymbol{r}_{j}\right)-(k-j) \alpha_{1}$ is one-dimensional for $0 \leq j \leq k$. Using the induction hypothesis on $k$, we know that the weight space of $\sum_{j=0}^{k} U\left(\mathfrak{n}^{-}\right) \mathbf{y}_{r_{j}} v_{r, s}$ of weight $\lambda-\operatorname{wt}\left(\boldsymbol{r}_{0}\right)-(k+1) \alpha_{1}$ has dimension $k+1$. Since the elements $\left(x_{\alpha_{1}}^{-}\right)^{j} \mathbf{y}_{\boldsymbol{r}_{k+1-j}} v_{r, s}$ for $0 \leq j \leq k+1$ are 
clearly linearly independent, it follows that $V\left(\lambda-\operatorname{wt}\left(\boldsymbol{r}_{k+1}\right)\right)$ is a submodule of $\sum_{j=0}^{k+1} U\left(\mathfrak{n}^{-}\right) \mathbf{y}_{r_{j}} v_{r, s}$.

Remark. Suppose $n>3$ and that $\lambda \in P^{+}$is such that $\operatorname{supp}(\lambda) \subseteq\{1,2,3, n\}$ with $\lambda\left(h_{n}\right)=1$. Since $R^{+}(n, 1,1)=R^{+}$, it follows that all of the above can be carried out and Proposition 5.7 remains valid (notice $d_{3}^{\prime}=1$ in this case).

5.8. Type D. Define the set $\mathscr{A}_{3}(\lambda)$ as in (5-2) and the maps wt and gr as in (5-4).

Proposition 5.9. If $\lambda \in P^{+}$is such that $\lambda\left(h_{i}\right)=0$ if $3<i<n-1$, then $M(\lambda) \cong T(\lambda)$.

(a) If $n=4$, then

$$
M(\lambda)[l] \cong \begin{cases}V\left(\lambda-l \theta_{1,1}\right) & \text { if } 0 \leq l \leq \lambda\left(h_{2}\right) \\ 0 & \text { otherwise }\end{cases}
$$

(b) If $n>4$, then

$$
M(\lambda)[l] \cong \bigoplus_{\boldsymbol{r} \in \mathscr{A}_{3}(\lambda): \operatorname{gr}(\boldsymbol{r})=l} V(\lambda-\mathrm{wt}(\boldsymbol{r})) .
$$

Proof. The proof is essentially the same as that of Proposition 5.7 using that $R^{+}\left(m \omega_{i}, 1\right)=R^{+}$if $i$ labels a spin node.

In particular, Proposition 5.9 gives the description of the graded characters of $M(\lambda)$ in types $D_{4}$ and $D_{5}$ for any $\lambda \in P^{+}$. If $\operatorname{supp}(\lambda)$ contains at most one of the spin nodes, it follows that Proposition 5.9 describes the character of the minimal affinizations of $V_{q}(\lambda)$ as well. Otherwise, it is just a lower bound.

Now, let $m \in \mathbb{Z}_{\geq 0}$ and set

$$
\mathscr{A}(m)=\left\{\boldsymbol{r}=\left(r_{1}, r_{2}, \ldots, r_{[(n-2) / 2]}\right) \in \mathbb{Z}_{\geq 0}^{[(n-2) / 2]}: m \geq r_{1} \geq \cdots \geq r_{[(n-2) / 2]}\right\} .
$$

Define

$$
\mathrm{wt}(\boldsymbol{r})=\sum_{j=1}^{[(n-2) / 2]} r_{j} \theta_{n-2 j, n-2 j} \quad \text { and } \quad \operatorname{gr}(\boldsymbol{r})=\sum_{j=1}^{[(n-2) / 2]} r_{j} .
$$

It was proved in [Chari 2001; CM 2006] that

$$
M\left(m \omega_{n-2}\right)[l]=\sum_{\boldsymbol{r} \in \mathscr{A}(m): \operatorname{gr}(\boldsymbol{r})=l} \mathbf{y}_{\boldsymbol{r}} v_{n-2, m} \cong \bigoplus_{\boldsymbol{r} \in \mathscr{A}(m): \operatorname{gr}(\boldsymbol{r})=l} V\left(m \omega_{n-2}-\mathrm{wt}(\boldsymbol{r})\right),
$$

where

$$
\mathbf{y}_{\boldsymbol{r}}=\prod_{j=1}^{[(n-2) / 2]}\left(x_{\theta_{n-2 j, n-2 j}, 1}^{-}\right)^{r_{j}} .
$$

Proceeding similarly to the proof of Proposition 5.7, one also proves the following. 
Proposition 5.10. Let $\lambda \in P^{+}$such that $\lambda\left(h_{i}\right)=0$ if $i<n-2$. Then $M(\lambda) \cong T(\lambda)$ and

$$
M(\lambda)[l] \cong \bigoplus_{\boldsymbol{r} \in \mathscr{A}\left(\lambda\left(h_{n-2}\right)\right): \operatorname{gr}(\boldsymbol{r})=l} V(\lambda-\mathrm{wt}(\boldsymbol{r})) .
$$

5.11. Multiple minimal affinizations: The regular case. Let $\mathfrak{g}$ be of types $D$ or $E$ and $i_{0} \in I$ be the unique node triply connected. Let also $J_{1}, J_{2}, J_{3} \subseteq I$ be an enumeration of the three maximal subdiagrams of type $A$ of the Dynkin diagram of $\mathfrak{g}$ (they are not admissible). Let also $J_{k}^{\prime}=J_{l} \cap J_{m}$ for $\{k, l, m\}=\{1,2,3\}$. It follows from [CP 1996b, Theorem 6.1] that, if $\lambda\left(h_{i_{0}}\right) \neq 0$ and $\lambda$ is supported on the three connected components of $I \backslash\left\{i_{0}\right\}$, then $V_{q}(\lambda)$ has exactly three equivalence classes of minimal affinizations. Moreover:

Theorem 5.12. Let $\lambda \in \mathscr{P}_{q}^{+}$be such that $\mathrm{wt}(\lambda)=\lambda$, where $\lambda$ is as above. Then $V_{q}(\lambda)$ is a minimal affinization of $V_{q}(\lambda)$ if and only if there exists $k \in\{1,2,3\}$ such that $V_{q}\left(\lambda_{J_{l}}\right)$ is a minimal affinization of $V_{q}\left(\lambda_{J_{l}}\right)$ for $l \neq k$.

Definition 5.13. Given $\lambda \in P^{+}$and $k \in\{1,2,3\}$, let $M_{k}(\lambda)$ be the quotient of $A(\lambda)$ by the submodule generated by the vectors $x_{\alpha, 1}^{-} v_{\lambda}$ for all $\alpha \in R_{J_{l}}^{+}$with $l \neq k$. Suppose $\lambda \in \mathscr{P}_{\mathrm{A}}^{++}$is such that $V_{q}\left(\lambda_{J_{l}}\right)$ is a minimal affinization of $V_{q}\left(\lambda_{J_{l}}\right)$ for $l \neq k$. Set $T_{k}(\lambda)$ to be the $\mathfrak{g}[t]$-submodule of $M\left(\lambda^{J_{k}^{\prime}}\right) \otimes L\left(\lambda^{I \backslash J_{k}^{\prime}}\right)$ generated by the top weight space.

It is easy to see that $M_{k}(\lambda)$ is a restricted $\mathfrak{g}[t]$-module and that $M(\lambda)$ is a quotient of $M_{k}(\lambda)$ for all $k$. Also, proceeding similarly to the proofs of Propositions 3.21 and 3.22 we get the following analogue (we omit the details).

Proposition 5.14. Let $\lambda \in \mathscr{P}_{\mathbb{A}}^{++}$and $k \in\{1,2,3\}$ be such that $V_{q}\left(\lambda_{J_{l}}\right)$ is a minimal affinization of $V_{q}\left(\lambda_{J_{l}}\right)$ for $l \neq k$. Then there exist surjective $\mathfrak{g}[t]$-module maps $M_{k}(\lambda) \rightarrow L(\lambda) \rightarrow T_{k}(\lambda)$.

Conjecture 5.15. Suppose $\lambda \in P^{+}$is supported on the three connected components of $I \backslash\left\{i_{0}\right\}$. Then, $T_{k}(\lambda)$ and $M_{k}(\lambda)$ are isomorphic for every $k \in\{1,2,3\}$.

Corollary 5.16. Suppose $\lambda \in \mathscr{P}_{\mathbb{A}}^{++}$and $k \in\{1,2,3\}$ are such that $V_{q}\left(\lambda_{J_{l}}\right)$ is a minimal affinization of $V_{q}\left(\lambda_{J_{l}}\right)$ for $l \neq k$ and $\mathrm{wt}(\lambda)$ is supported on the three connected components of $I \backslash\left\{i_{0}\right\}$. Then, $T_{k}(\lambda) \cong L(\lambda) \cong M_{k}(\lambda)$.

We now prove Conjecture 5.15 for $\mathfrak{g}$ of type $D_{4}$. Thus, let $\lambda \in P^{+}$be such that $\lambda\left(h_{i}\right) \neq 0$ for all $i \neq 2$, and let $\lambda$ be such that $V_{q}(\lambda)$ is a minimal affinization of $V_{q}(\lambda)$. Set also $J_{1}=\{1,2,3\}, J_{2}=\{1,2,4\}$, and $J_{3}=\{2,3,4\}$. Without loss of generality we can assume that $V_{q}\left(\lambda_{J_{1}}\right)$ and $V_{q}\left(\lambda_{J_{2}}\right)$ are minimal affinizations. We want to show that $T_{3}(\lambda) \cong M_{3}(\lambda)$ in this case. We also assume that

$$
\boldsymbol{\lambda}=\boldsymbol{\omega}_{1, a, \lambda\left(h_{1}\right)} \boldsymbol{\omega}_{2, a q^{\lambda\left(h_{1}\right)+\lambda\left(h_{2}\right)+1}, \lambda\left(h_{2}\right)} \boldsymbol{\omega}_{3, a q^{\lambda\left(h_{2}\right)+\lambda\left(h_{3}\right)+1}, \lambda\left(h_{3}\right)} \boldsymbol{\omega}_{4, a q^{\lambda\left(h_{2}\right)+\lambda\left(h_{4}\right)+1}, \lambda\left(h_{4}\right)}
$$


for some $a \in \mathbb{C}^{\times}$. The case

$$
\boldsymbol{\lambda}=\boldsymbol{\omega}_{1, a, \lambda\left(h_{1}\right)} \boldsymbol{\omega}_{2, a q^{-\left(\lambda\left(h_{1}\right)+\lambda\left(h_{2}\right)+1\right)}, \lambda\left(h_{2}\right)} \boldsymbol{\omega}_{3, a q^{-\left(\lambda\left(h_{2}\right)+\lambda\left(h_{3}\right)+1\right)}, \lambda\left(h_{3}\right)} \boldsymbol{\omega}_{4, a q^{-\left(\lambda\left(h_{2}\right)+\lambda\left(h_{4}\right)+1\right)}, \lambda\left(h_{4}\right)}
$$

is proved similarly. If $\lambda\left(h_{2}\right) \neq 0$, these two cases cover all minimal affinizations such that $V_{q}\left(\lambda_{J_{1}}\right)$ and $V_{q}\left(\lambda_{J_{2}}\right)$ are also minimal affinizations. Otherwise, there are two more possibilities for $\lambda$ (see the closing remark of Section 5.18).

Let $v$ be the image of 1 in $M_{3}(\lambda)$. By the definition of $M_{3}(\lambda)$, we have the relations

$$
x_{\alpha_{i}, 1}^{-} v=x_{\alpha_{2}+\alpha_{j}, 1}^{-} v=x_{\alpha_{1}+\alpha_{2}+\alpha_{3}, 1}^{-} v=x_{\alpha_{1}+\alpha_{2}+\alpha_{4}, 1}^{-} v=0
$$

for all $i, j \in I$ and $j \neq 2$. Using the commutation relations $\left[x_{\alpha}^{-}, x_{\beta}^{-}\right]=x_{\alpha+\beta}^{-}$(up to multiple) we also get

$$
x_{\alpha, 2}^{-} v=0 \quad \text { for all } \alpha \in R^{+}
$$

Let $\vartheta_{1}=\sum_{i=1}^{4} \alpha_{i}=\omega_{1}+\omega_{3}+\omega_{4}-\omega_{2}, \vartheta_{2}=\vartheta_{1}-\alpha_{1}=\omega_{3}+\omega_{4}-\omega_{1}$, and $\theta=\theta_{1,1}=\vartheta_{1}+\alpha_{2}=\omega_{2}$. It follows that

$$
M_{3}(\lambda)=\sum_{\boldsymbol{r} \in \mathbb{Z}_{\geq 0}^{3}} U\left(\mathfrak{n}^{-}\right) \mathbf{y}_{\boldsymbol{r}} v, \quad \text { where } \mathbf{y}_{\boldsymbol{r}}=\left(x_{\theta, 1}^{-}\right)^{r_{3}}\left(x_{\vartheta_{2}, 1}^{-}\right)^{r_{2}}\left(x_{\vartheta_{1}, 1}^{-}\right)^{r_{1}} .
$$

Since $\left\{\vartheta_{1}, \vartheta_{2}, \theta\right\}$ is a linearly independent subset of $\mathfrak{h}^{*}$, it follows as before that $m_{\mu}\left(M_{3}(\lambda)\right) \leq 1$ for every $\mu \in P^{+}$, with equality only if $\mu=\lambda-r_{1} \vartheta_{1}-r_{2} \vartheta_{2}-r_{3} \theta$ for some $r_{j} \in \mathbb{Z}_{\geq 0}$. But such elements are dominant if and only if

$$
r_{1} \leq \lambda\left(h_{1}\right)+r_{2}, \quad r_{3} \leq \lambda\left(h_{2}\right)+r_{1}, \quad r_{1}+r_{2} \leq \min \left\{\lambda\left(h_{3}\right), \lambda\left(h_{4}\right)\right\} .
$$

Set

$$
\mathscr{D}_{3}(\lambda)=\left\{\boldsymbol{r} \in \mathbb{Z}_{\geq 0}^{3}: r_{1} \leq \lambda\left(h_{1}\right), r_{3} \leq \lambda\left(h_{2}\right), r_{1}+r_{2} \leq \min \left\{\lambda\left(h_{3}\right), \lambda\left(h_{4}\right)\right\}\right\} .
$$

Proceeding similarly to the proof of Lemma 5.6, one proves that

$$
M_{3}(\lambda)=\sum_{r \in \mathscr{D}_{3}(\lambda)} U\left(\mathfrak{n}^{-}\right) \mathbf{y}_{\boldsymbol{r}} v
$$

Given $\boldsymbol{r} \in \mathbb{Z}_{\geq 0}^{3}$, define

$$
\mathrm{wt}(\boldsymbol{r})=r_{1} \vartheta_{1}+r_{2} \vartheta_{2}+r_{3} \theta \text { and } \operatorname{gr}(\boldsymbol{r})=r_{1}+r_{2}+r_{3} .
$$

Since $\left\{\vartheta_{1}, \vartheta_{2}, \theta\right\}$ is linearly independent, it follows that wt is an injective function. To complete the proof of Conjecture 5.15 in this case, it suffices to prove that $m_{\mu}\left(T_{3}(\lambda)\right) \geq 1$ if $\mu=\lambda-\operatorname{wt}(\boldsymbol{r})$ for some $\boldsymbol{r} \in \mathscr{D}_{3}(\lambda)$. In particular, it will follow 
that

$$
M_{3}(\lambda)[l]=\bigoplus_{\boldsymbol{r} \in \mathscr{D}_{3}(\lambda): \operatorname{gr}(\boldsymbol{r})=l} V(\lambda-\mathrm{wt}(\boldsymbol{r})) .
$$

Proposition 5.17. Let

$$
b \in \mathbb{A}^{\times}, \quad \mu=m_{3} \omega_{3}+m_{4} \omega_{4} \in P^{+}, \quad \boldsymbol{\mu}=\boldsymbol{\omega}_{3, b, m_{3}} \boldsymbol{\omega}_{4, b q^{m_{4}-m_{3}, m_{4}} .} .
$$

Then, $L(\boldsymbol{\mu})[l] \cong V\left(\mu-l \vartheta_{2}\right)$ for $0 \leq l \leq \min \left\{m_{3}, m_{4}\right\}$ and $L(\boldsymbol{\mu})[l]=0$ otherwise. Proof. Let $v$ be a highest-weight vector of $L(\boldsymbol{\mu})$. Clearly $v$ satisfies relations (5-6) and (5-7). Moreover, proceeding as above, we get

$$
L(\boldsymbol{\mu})=\sum_{\boldsymbol{r} \in \mathscr{D}_{3}(\mu)} U(\mathfrak{g}) \mathbf{y}_{\boldsymbol{r}} v=\bigoplus_{r=0}^{\min \left\{m_{3}, m_{4}\right\}} U\left(\mathfrak{n}^{-}\right)\left(x_{\vartheta_{2}, 1}^{-}\right)^{r} v
$$

and, by Lemma 5.2 once more,

$$
\left(x_{\vartheta_{1}, 1}^{-}\right)^{r_{1}}\left(x_{\vartheta_{2}, 1}^{-}\right)^{r_{2}} v=\left(x_{\alpha_{1}}^{-}\right)^{r_{1}}\left(x_{\vartheta_{2}, 1}^{-}\right)^{r_{1}+r_{2}} v .
$$

Without loss of generality, assume $m_{4} \geq m_{3} \geq 1$ and observe that

$$
\boldsymbol{\mu}=\left(\prod_{j=0}^{m_{3}-1} \boldsymbol{\omega}_{\omega_{3}+\omega_{4}, b q^{1-m_{3}+2 j}}\right)\left(\prod_{j=0}^{m_{4}-m_{3}-1} \boldsymbol{\omega}_{4, b q^{m_{3}+1+2 j}}\right) .
$$

Then by Proposition 4.3 and its corollary, $V_{q}(\boldsymbol{\mu})$ is the $U_{q}(\tilde{\mathfrak{g}})$-submodule of

$$
\begin{aligned}
& \left(V_{q}\left(\boldsymbol{\omega}_{\omega_{3}+\omega_{4}, b q^{1-m_{3}}}\right) \otimes \cdots \otimes V_{q}\left(\boldsymbol{\omega}_{\omega_{3}+\omega_{4}, b q^{m_{3}-1}}\right)\right) \\
& \quad \otimes\left(V_{q}\left(\boldsymbol{\omega}_{4, b q^{m_{3}+1}}\right) \otimes \cdots \otimes V_{q}\left(\boldsymbol{\omega}_{4, b q^{2 m_{4}-m_{3}-1}}\right)\right)
\end{aligned}
$$

that is generated by the top weight space. Let $M^{\prime}\left(\omega_{3}+\omega_{4}\right)$ be the pullback of $\overline{V_{q}\left(\omega_{\omega_{3}+\omega_{4}, b q^{m}}\right)}$ by $\tau_{b}$, where $m \in \mathbb{Z}$, and let $T^{\prime}(\mu)$ be the $\mathfrak{g}[t]$-submodule of $M^{\prime}\left(\omega_{3}+\omega_{4}\right)^{\otimes m_{3}} \otimes M\left(\omega_{4}\right)^{\otimes m_{4}-m_{3}-1}$. As before, it follows from Lemma 2.20 that $T^{\prime}(\mu)$ is a quotient of $L(\mu)$. Hence, we are left to show that $T^{\prime}(\mu)$ has $V\left(\mu-l \vartheta_{2}\right)$ as an irreducible $\mathfrak{g}$-submodule for every $0 \leq l \leq m_{3}$. Moreover, it suffices to consider the case $m_{4}=m_{3}=m \in \mathbb{Z}_{>0}$. Observe that $V_{q}\left(\omega_{\omega_{3}+\omega_{4}, b q^{m}}\right)$ is not a minimal affinization and that $V\left(\omega_{3}\right) \otimes V\left(\omega_{4}\right) \cong V\left(\omega_{3}+\omega_{4}\right) \oplus V\left(\omega_{1}\right)$. In other words, the proposition is proved for $m_{3}=m_{4}=1$. Finally, let $v_{j}$ for $j=1, \ldots, m$ be a highest-weight vector of the $j$-th copy of $M^{\prime}\left(\omega_{3}+\omega_{4}\right)$ in $M^{\prime}\left(\omega_{3}+\omega_{4}\right)^{\otimes m}$, and let $v_{j}^{0}$ be its image in $M^{\prime}\left(\omega_{3}+\omega_{4}\right)(0)$. Then

$$
\begin{aligned}
\left(x_{\vartheta_{2}, 1}^{-}\right)^{l}\left(v_{1} \otimes v_{2} \otimes \cdots \otimes\right. & \left.v_{l} \otimes v_{l+1}^{0} \otimes \cdots \otimes v_{m}^{0}\right) \\
& =\left(x_{\vartheta_{2}, 1}^{-} v_{1}\right) \otimes\left(x_{\vartheta_{2}, 1}^{-} v_{2}\right) \otimes \cdots \otimes\left(x_{\vartheta_{2}, 1}^{-} v_{l}\right) \otimes v_{l+1}^{0} \otimes \cdots \otimes v_{m}^{0}
\end{aligned}
$$

and we are done using a simple induction on $l$. 
Let $v_{1}$ be a highest-weight vector of $M\left(\lambda^{\{1,2\}}\right)$, and let $v_{2}$ be a highest-weight vector of $L\left(\lambda^{\{3,4\}}\right)$. It follows from Proposition 5.9 and (5-11) that if $r \in \mathscr{D}_{3}(\lambda)$, then

$$
\mathbf{y}_{\boldsymbol{r}}\left(v_{1} \otimes v_{2}\right)=\left(\left(x_{\theta, 1}^{-}\right)^{r_{3}} v_{1}\right) \otimes\left(\left(x_{\alpha_{1}}^{-}\right)^{r_{1}}\left(x_{\vartheta_{2}, 1}^{-}\right)^{r_{1}+r_{2}} v_{2}\right) .
$$

The proof of (5-10) is completed similarly to the end of the proof of Proposition 5.7.

5.18. Multiple minimal affinizations: The irregular case. Keep the notation of Section 5.11. If $\lambda$ is supported on the three connected components of $I \backslash\left\{i_{0}\right\}$ and $\lambda\left(h_{i_{0}}\right)=0$, it follows from [CP 1996a] that the number of equivalence classes of minimal affinizations of $V_{q}(\lambda)$ is not uniformly bounded (it grows as $\lambda$ "grows"). If $\mathfrak{g}$ is of type $E$, write $I$ as the disjoint union of two connected subdiagrams of type $A$, say $I_{1}$ and $I_{2}$, and the subdiagram of type $D_{4}$, say $J$. For $\mathfrak{g}$ of type $D$ we write $I$ as the disjoint union of a subdiagram $I_{1}$ of type $A$ and the subdiagram $J$ of type $D_{4}$ (for convenience we set $I_{2}=\varnothing$ and $\lambda^{\varnothing}=0$ ). Similarly to the proof of Proposition 3.21, one proves this:

Proposition 5.19. Suppose $\lambda \in P^{+}$and $\lambda \in \mathscr{P}_{q}^{+}$are such that $V_{q}(\lambda)$ is a minimal affinization of $V_{q}(\lambda)$. Then $L(\lambda)$ projects onto the $\mathfrak{g}[t]$-submodule of $L\left(\lambda^{I_{1}}\right) \otimes$ $L\left(\lambda^{J}\right) \otimes L\left(\lambda^{I_{2}}\right)$ generated by the top weight space.

We then have a natural conjecture, the discussion of which we postpone to a forthcoming publication.

Conjecture 5.20. Suppose $\lambda \in P^{+}$and $\lambda \in \mathscr{P}_{q}^{+}$are such that $V_{q}(\lambda)$ is a minimal affinization of $V_{q}(\lambda)$. Then $L(\lambda)$ is isomorphic to the $\mathfrak{g}[t]$-submodule of $L\left(\lambda^{I_{1}}\right) \otimes$ $L\left(\lambda^{J}\right) \otimes L\left(\lambda^{I_{2}}\right)$ generated by the top weight space.

Remark. Let $\lambda$ be as in [CP 1996a, Theorem $2.2(a)_{3,4}$ or (b) $)_{3,4}$ ]. If conditions (a) $)_{3,4}$ are satisfied, the results of Section 5.11 apply, and hence the graded character of $L(\lambda)$ is given by the right side of (5-10). To prove the conjecture of remark (1) following that theorem, it suffices to show that if conditions (b) $)_{3,4}$ are satisfied, then the graded character of $L(\lambda)$ is also given by the right side of (5-10). The proof is essentially the same as for the former case replacing Proposition 5.17 by its appropriate obvious modification. We omit the details.

\section{Acknowledgment}

I thank D. Jakelić for helpful discussions.

\section{References}

[Beck 1994] J. Beck, "Braid group action and quantum affine algebras", Comm. Math. Phys. 165:3 (1994), 555-568. MR 95i:17011 Zbl 0807.17013 
[Beck et al. 1999] J. Beck, V. Chari, and A. Pressley, "An algebraic characterization of the affine canonical basis”, Duke Math. J. 99:3 (1999), 455-487. MR 2000g:17013 Zbl 0964.17013

[Chari 1986] V. Chari, "Integrable representations of affine Lie algebras", Invent. Math. 85:2 (1986), 317-335. MR 88a:17034 Zbl 0603.17011

[Chari 1995] V. Chari, "Minimal affinizations of representations of quantum groups: The rank 2 case”, Publ. Res. Inst. Math. Sci. 31:5 (1995), 873-911. MR 96m:17021 Zbl 0855.17010

[Chari 2001] V. Chari, "On the fermionic formula and the Kirillov-Reshetikhin conjecture", Internat. Math. Res. Notices 12 (2001), 629-654. MR 2002i:17019 Zbl 0982.17004

[Chari 2002] V. Chari, "Braid group actions and tensor products", Int. Math. Res. Not. 7 (2002), 357-382. MR 2003a:17014 Zbl 0990.17009

[Chari and Greenstein 2009] V. Chari and J. Greenstein, "A family of Koszul algebras arising from finite-dimensional representations of simple Lie algebras", Adv. Math. 220:4 (2009), 1193-1221. MR 2483719 Zbl 1165.17005

[Chari and Hernandez 2008] V. Chari and D. Hernandez, "Beyond Kirillov-Reshetikhin modules", preprint, 2008. arXiv 0812.1716

[CM 2005] V. Chari and A. A. Moura, "Characters and blocks for finite-dimensional representations of quantum affine algebras”, Int. Math. Res. Not. 2005:5 (2005), 257-298. MR 2006a:17021 Zbl 1074.17004

[CM 2006] V. Chari and A. Moura, "The restricted Kirillov-Reshetikhin modules for the current and twisted current algebras", Comm. Math. Phys. 266:2 (2006), 431-454. MR 2007m:17035 Zbl 1118.17007

[CM 2007] V. Chari and A. Moura, "Kirillov-Reshetikhin modules associated to $G_{2}$ ", pp. 4159 in Lie algebras, vertex operator algebras and their applications, edited by Y.-Z. Huang and K. C. Misra, Contemp. Math. 442, Amer. Math. Soc., Providence, RI, 2007. MR 2009i:17036 Zbl 05263351

[CP 1986] V. Chari and A. Pressley, "New unitary representations of loop groups", Math. Ann. 275:1 (1986), 87-104. MR 88f:17029 Zbl 0603.17012

[CP 1994a] V. Chari and A. Pressley, A guide to quantum groups, Cambridge University Press, 1994. MR 95j:17010 Zbl 0839.17009

[CP 1994b] V. Chari and A. Pressley, "Small representations of quantum affine algebras", Lett. Math. Phys. 30:2 (1994), 131-145. MR 95b:17017 Zbl 0795.17008

[CP 1995] V. Chari and A. Pressley, "Minimal affinizations of representations of quantum groups: The nonsimply-laced case”, Lett. Math. Phys. 35:2 (1995), 99-114. MR 96g:17012 Zbl 0855.17011 [CP 1996a] V. Chari and A. Pressley, "Minimal affinizations of representations of quantum groups: The irregular case”, Lett. Math. Phys. 36:3 (1996), 247-266. MR 96m:17022 Zbl 0857.17011

[CP 1996b] V. Chari and A. Pressley, "Minimal affinizations of representations of quantum groups: The simply laced case”, J. Algebra 184:1 (1996), 1-30. MR 97f:17012 Zbl 0893.17010

[CP 1997] V. Chari and A. Pressley, "Quantum affine algebras at roots of unity", Represent. Theory 1 (1997), 280-328. MR 98e:17018 Zbl 0891.17013

[CP 2001] V. Chari and A. Pressley, "Weyl modules for classical and quantum affine algebras", Represent. Theory 5 (2001), 191-223. MR 2002g:17027 Zbl 0989.17019

[Damiani 1998] I. Damiani, "La $R$-matrice pour les algèbres quantiques de type affine non tordu", Ann. Sci. École Norm. Sup. (4) 31:4 (1998), 493-523. MR 99g:17027 Zbl 0911.17005

[Frenkel and Mukhin 2001] E. Frenkel and E. Mukhin, "Combinatorics of $q$-characters of finitedimensional representations of quantum affine algebras", Comm. Math. Phys. 216:1 (2001), 23-57. MR 2002c:17023 Zbl 1051.17013 
[Frenkel and Reshetikhin 1999] E. Frenkel and N. Reshetikhin, "The $q$-characters of representations of quantum affine algebras and deformations of W-algebras", pp. 163-205 in Recent developments in quantum affine algebras and related topics (Raleigh, NC, 1998), edited by N. Jing and K. C. Misra, Contemp. Math. 248, Amer. Math. Soc., Providence, RI, 1999. MR 2002f:17022 Zbl 0973.17015

[Hernandez 2007] D. Hernandez, "On minimal affinizations of representations of quantum groups", Comm. Math. Phys. 276:1 (2007), 221-259. MR 2008h:17014 Zbl 1141.17011

[Humphreys 1972] J. E. Humphreys, Introduction to Lie algebras and representation theory, Graduate Texts in Mathematics 9, Springer, New York, 1972. MR 48 \#2197 Zbl 0254.17004

[Jakelić and Moura 2009a] D. Jakelić and A. Moura, "Finite-dimensional representations of hyper loop algebras over non-algebraicaly closed fields", Algebras and Representation Theory (2009).

[Jakelić and Moura 2009b] D. Jakelić and A. Moura, "Tensor products, characters, and blocks of finite-dimensional representations of quantum affine algebras at roots of unity”, preprint, 2009. arXiv 0909.2198

[Jimbo 1986] M. Jimbo, "A $q$-analogue of $U(\mathfrak{g l}(N+1))$, Hecke algebra, and the Yang-Baxter equation”, Lett. Math. Phys. 11:3 (1986), 247-252. MR 87k:17011 Zbl 0602.17005

[Kassel 1995] C. Kassel, Quantum groups, Graduate Texts in Mathematics 155, Springer, New York, 1995. MR 96e:17041 Zbl 0808.17003

[Kirillov and Reshetikhin 1987] A. N. Kirillov and N. Y. Reshetikhin, "Representations of Yangians and multiplicities of the inclusion of the irreducible components of the tensor product of representations of simple Lie algebras", Zap. Nauchn. Sem. Leningrad. Otdel. Mat. Inst. Steklov. 160 (1987), 211-221. In Russian; translated in J. Sov. Math. 52:3 (1990), 3156-3164. MR 89b:17012 Zbl 0637.16007

[Lusztig 1993] G. Lusztig, Introduction to quantum groups, Progress in Mathematics 110, Birkhäuser, Boston, MA, 1993. MR 94m:17016 Zbl 0788.17010

[Nakai and Nakanishi 2007a] W. Nakai and T. Nakanishi, "Paths and tableaux descriptions of JacobiTrudi determinant associated with quantum affine algebra of type $C_{n}$ ", SIGMA Symmetry Integrability Geom. Methods Appl. 3 (2007), paper 078. MR 2008f:17030 Zbl 1142.17009

[Nakai and Nakanishi 2007b] W. Nakai and T. Nakanishi, "Paths and tableaux descriptions of JacobiTrudi determinant associated with quantum affine algebra of type $D_{n}$ ", J. Algebraic Combin. 26:2 (2007), 253-290. MR 2008e:17013 Zbl 05204341

[Nakai and Nakanishi 2008] W. Nakai and T. Nakanishi, "On Frenkel-Mukhin algorithm for qcharacter of quantum affine algebras”, preprint, version 2, 2008. arXiv 0801.2239v2

Received January 28, 2009. Revised July 19, 2009.

\author{
ADRIANO MOURA \\ UNICAMP - IMECC \\ DEPARTAMENTO DE MATEMÁtiCA \\ 13083-859 CAMPINAS-SP \\ BRAZIL \\ aamoura@ime.unicamp.br \\ http://www.ime.unicamp.br/ aamoura
}

